\title{
An Improved Scale-Adaptive Simulation Model for Massively Separated Flows
}

\author{
Yue Liu, Xiaorong Guan (D), and Cheng Xu \\ School of Mechanical Engineering, Nanjing University of Science and Technology, Nanjing 210094, China \\ Correspondence should be addressed to Xiaorong Guan; gxr@njust.edu.cn
}

Received 30 November 2017; Accepted 31 January 2018; Published 22 March 2018

Academic Editor: William W. Liou

Copyright ( 2018 Yue Liu et al. This is an open access article distributed under the Creative Commons Attribution License, which permits unrestricted use, distribution, and reproduction in any medium, provided the original work is properly cited.

\begin{abstract}
A new hybrid modelling method termed improved scale-adaptive simulation (ISAS) is proposed by introducing the von Karman operator into the dissipation term of the turbulence scale equation, proper derivation as well as constant calibration of which is presented, and the typical circular cylinder flow at $\mathrm{Re}=3900$ is selected for validation. As expected, the proposed ISAS approach with the concept of scale-adaptive appears more efficient than the original SAS method in obtaining a convergent resolution, meanwhile, comparable with DES in visually capturing the fine-scale unsteadiness. Furthermore, the grid sensitivity issue of DES is encouragingly remedied benefiting from the local-adjusted limiter. The ISAS simulation turns out to attractively represent the development of the shear layers and the flow profiles of the recirculation region, and thus, the focused statistical quantities such as the recirculation length and drag coefficient are closer to the available measurements than DES and SAS outputs. In general, the new modelling method, combining the features of DES and SAS concepts, is capable to simulate turbulent structures down to the grid limit in a simple and effective way, which is practically valuable for engineering flows.
\end{abstract}

\section{Introduction}

Large-scale separations coupled with chaotic, nonlinear phenomena are often strongly pronounced in aerodynamic and industrial turbulent flows; the wide investigations for accurately predicting such flows have proven one of the most challenging CFD tasks, which is mainly related to the complex boundary layer and the troublesome broad turbulence spectra $[1,2]$. Thus, at least a partial resolution of the turbulence spectrum has been the main topic of turbulence modelling for technical simulations in the last decades. In recent years, the hybrid RANS/LES methods are of great interest to both academic institutions and industry applications with the motivation that it is powerless to solve the problems at hand when using the LES or RANS method alone [3-5]. As the continuing theoretical progress and from a practical standpoint, many scholars recognize that whether a turbulence model can predict turbulent structures relies on the available eddy viscosity level when using the eddy viscous method. Following the LES method, a typical hybrid LES/ RANS model termed detached eddy simulation (DES) was firstly proposed by Spalart et al. [6]. It is well known that the DES concept combines the advantages of RANS and LES methods in a simple and effective way by using the grid spacing as an adjusting scale, which has been proven promising for engineering flows, and a variety of hybrid approaches have since appeared $[3,7]$. However, the DES models are not without problems $[8,9]$, and the main stumbling stone for DES is the ambiguous response to the grid refinement. When exceeding a critical level of grid refinement near walls, the standard DES formulation may negatively impact the RANS performance and arise some issues, such as, gray area, modeled stress depletion (MSD), and grid-induced separation (GIS) [10]. Various remedial measures such as DDES $[11,12]$, IDDES [13-15], and zonal DES [16] have been conducted to protect the boundary layer from the DES limiter; nevertheless, the remedies do not entirely avoid the issues appearing either conservative or inefficient $[10,17]$.

In contrast to DES which employs grid size as the deciding scale to produce LES content for unstable flows, the concept of scale-adaptive simulation (SAS) characterized with a second-order von Karman length scale $\left(L_{\mathrm{vk}}\right)$ was firstly conducted by Menter et al. [18]. The higher derivative reflects the local flow physics and gradually adjusts the resolution to 
the resolved scales with grid independency, which is thereby viewed the main advantage of SAS over the other hybrid methods. Again because of grid independence, the SAS is often treated as an advanced unsteady RANS (URANS) [3]. Since the first one-equation KE1E-SAS was introduced, several transformations based on different RANS background have been conducted by the Menter team [19-21]. In recent years, the SAS models have been extensively validated in numerous test cases and continuously undergone certain evolution, which dramatically shows its potential in the prediction of actual turbulent flows [22-24].

At this point, two appealing hybrid methods regarding DES and SAS have been briefly reviewed. It was expected that the LES solutions are still too expensive for industrial flows with high Reynolds number while the feasible RANS/LES approaches will dominate turbulence modelling in the next few decades $[10,17]$; thus, the modification and improvement for the existing hybrid methods are very essential. For this perspective, we naturally intend to combine the formulations of DES and SAS in this paper. On one hand, the simple modelling mechanism using the ratio of modelled scale to grid scale enables DES method to be the most widely used hybrid method. But the strict grid requirement of which is difficult to cover the entire boundary layer [25]. On the other hand, the $L_{\mathrm{vk}}$ scale with concept of scale-adaptive enables the solution smoothly varying from RANS region to LES-like region, which allows easier grid generation especially in the complex applications. Nevertheless, the current SAS mechanism by an additional source term is troublesome and may be less efficient to obtain a convergent resolution. Furthermore, the von Karman scale and grid scale are proportional to the shear layer thickness at near-wall region, while at the separated region, both of them are proportional to the resolved scales and responsible for the generation of spectral content with LES-level viscosity $[21,26]$. It thereby means the two scales are theoretically compatible with each other. In fact, there have been several reformulations by introducing $L_{\mathrm{vk}}$ scale into the one-equation SA-DES model $[27,28]$, while an even more interesting example is the "turbulenceresolving RANS" (TRANS) approach based on the twoequation $k-\omega$ model [29]. The TRANS model uses the ratio between strain and vorticity $(S / \Omega)$ as the spatial operator rather than the high derivative, which also allows the reproduction of unsteady content and makes SAS more intuitive, so the model is practical but without a clear physical mechanism between the eddy viscosity and resolved scales.

In this paper, a new hybrid model named improved scaleadaptive simulation (ISAS) is proposed by replacing the grid scale of the DES with the von Karman scale from SAS. The derivation and the constant calibration of the ISAS are described in the following sections. As for the model validation, the typical bluff body flow over a circular cylinder at $\mathrm{Re}=3900$ was selected mainly due to the rich flow physics and various references regarding both experimental reports and numerical studies. The ISAS performance was tested by numerical comparisons with available experimental data on both fine and coarse grids, and the main objective is to verify the capability of the underlying method in predicting the massively separated flows. It is worth noting that all the hybrid formulations for the following simulations are based on the two-equation shear stress transport (SST) model [30], which is demonstrated in robustly and accurately predicting the boundary layers with adverse pressure gradients.

\section{Turbulence Modelling}

2.1. SST Model Formulation. Benefiting from $k-\omega$ model in calculating the viscous sublayer as well as $k-\varepsilon$ model in better dealing with the fully turbulent flows away from walls, Menter's two-equation SST model combines the best features of standard $k$ - $\omega$ and $k-\varepsilon$ models, which is very popular in the prediction of aeronautics flows and treated as a main platform for the hybrid formulations. In this paper, the target SST model is the version of Menter [31], and the two independent transport equations of which are written as follows:

$$
\begin{aligned}
\frac{\partial \rho k}{\partial t}+\nabla \cdot(\rho \mathbf{U} k)= & \tilde{P}_{k}-\beta^{*} \rho k \omega+\nabla \cdot\left[\left(\mu+\sigma_{k} \mu_{t}\right) \nabla k\right], \\
\frac{\partial \rho \omega}{\partial t}+\nabla \cdot(\rho \mathbf{U} \omega)= & \frac{\alpha}{v_{t}} \tilde{P}_{k}-\beta \rho \omega^{2}+\nabla \cdot\left[\left(\mu+\sigma_{\omega} \mu_{t}\right) \nabla \omega\right] \\
& +\left(1-F_{1}\right) \frac{2 \rho \sigma_{\omega_{2}}}{\omega} \nabla k \nabla \omega,
\end{aligned}
$$

where $k$ is the turbulence kinetic energy, $\omega$ is the specific dissipation rate, $\rho$ is the density, $\mathbf{U}$ is the velocity vector, and $\mu$ is the dynamic viscosity.

All model parameters $\varphi$ are obtained by an applicable compromise of $k-\omega$ and $k$ - $\varepsilon$ constants via $\varphi=F_{1} \varphi_{1}+$ $\left(1-F_{1}\right) \varphi_{2}$, in which the blending function $F_{1}$ is defined as

$$
F_{1}=\tanh \left\{\left\{\min \left[\max \left(\frac{\sqrt{k}}{\beta^{*} \omega d}, \frac{500 \mu}{d^{2} \rho \omega}\right), \frac{4 \rho \sigma_{\omega_{2}} k}{\mathrm{CD}_{k \omega} d^{2}}\right]\right\}^{4}\right\}
$$

with $\mathrm{CD}_{k \omega}=\max \left(2 \rho \sigma_{\omega_{2}}(1 / \omega)\left(\partial k / \partial x_{i}\right)\left(\partial \omega / \partial x_{i}\right), 10^{-10}\right)$ and $d$ is the distance from the nearest solid surface. The adopted model constants are $\beta^{*}=0.09, \alpha_{1}=0.5556, \alpha_{2}=0.44$, $\beta_{1}=0.075, \beta_{2}=0.0828, \sigma_{k_{1}}=0.85, \sigma_{k_{2}}=1.0, \sigma_{\omega_{1}}=0.5$, and $\sigma_{\omega_{2}}=0.856$.

The turbulent eddy viscosity is expressed as

$$
\mu_{t}=\frac{\alpha_{1} \rho k}{\max \left(\alpha_{1} \omega, S F_{2}\right)}
$$

where $S$ is the scalar invariant of the strain rate $\left(S_{i j}\right)$, $S=\sqrt{2 S_{i j} S_{i j}}, \alpha_{1}=0.31$, and $F_{2}$ is a second blending function defined as

$$
F_{2}=\tanh \left\{\left[\max \left(\frac{2 \sqrt{k}}{\beta^{*} \omega d}, \frac{500 \mu}{d^{2} \rho \omega}\right)\right]^{2}\right\}
$$

It is important to note that a limit function is used in recent production terms to fairly avoid the build-up issue 
in stagnation regions. The production terms are expressed as follows:

$$
\begin{aligned}
& P_{k}=\mu_{t} S^{2}, \\
& \tilde{P}_{k}=\min \left(P_{k}, 10 \times \beta^{*} \rho k \omega\right) .
\end{aligned}
$$

2.2. DES Model Formulation. The transformation of the DES term to the SST model is firstly introduced by Strelets [32] and simplified by Menter et al. [17]. Here, the SST-DES model of Menter is viewed as the standard DES formulation, and the distinguishing factor over the background SST model lies in a hybrid function $\left(F_{\mathrm{DES}}\right)$ directly acting on the dissipation term of the $k$-scale equation, which is expressed as

$$
\beta^{*} \rho k \omega \rightarrow \beta^{*} \rho k \omega \times F_{\mathrm{DES}}, \quad F_{\mathrm{DES}}=\max \left(\frac{l_{k \omega}}{C_{\mathrm{DES}} \Delta}, 1.0\right),
$$

where $C_{\mathrm{DES}}$ is the model constant and is equal to 0.61 , as the limiter should only be active in the $k-\varepsilon$ region, $l_{k \omega}$ is the turbulent length scale defined by $l_{k \omega}=\sqrt{k} /\left(\beta^{*} \omega\right)$, and $\Delta$ is the local grid scale reading $\Delta=\max (\Delta x, \Delta y, \Delta z)$.

As shown above, in DES, the switch from RANS to LESlike modes depends on the ratio between the turbulent length scale (modelled) and grid scale (resolved). When near walls, $l_{k \omega}<C_{\mathrm{DES}} \Delta$ and $F_{\mathrm{DES}}=1.0$, the model function is passive and therefore suitable for the stable flows in a RANS mode, whereas, when typically in the separating regions with refinement grids, $l_{k \omega}>C_{\mathrm{DES}} \Delta$ and $F_{\mathrm{DES}}>1.0$, a LES-like mode is gained.

2.3. SAS Model Formulation. The formulation of the SSTSAS model differs from the pure SST model by the additional source term $\left(Q_{\mathrm{SAS}}\right)$ in the $\omega$-scale equation [20]:

$Q_{\mathrm{SAS}}=\max \left[\rho \xi \kappa S^{2}\left(\frac{L_{k \omega}}{L_{\mathrm{vk}}}\right)^{2}-C \cdot \frac{2 \rho k}{\sigma_{\varphi}} \max \left(\frac{|\nabla \omega|^{2}}{\omega^{2}}, \frac{|\nabla k|^{2}}{k^{2}}\right), 0\right]$,

where similar to DES, $L_{k \omega}$ is the modelled turbulence length scale and reads $L_{k \omega}=\sqrt{k} /\left(C_{\mu}^{1 / 4} \omega\right)$, while the distinctive von Karman length scale $\left(L_{\mathrm{vk}}\right)$ is achieved by $L_{v k}=\kappa S /\left|\nabla^{2} U\right|$. The model constants are $C=2.0, \xi=3.51, \kappa=0.41, \sigma \varphi=$ 0.67 , and $C_{\mu}=0.09$.

It is worth mentioning that the second derivative $\left(U^{\prime \prime}\right)$ is used instead of the third derivative, appearing more sufficient in the 3-D simulation [21, 22, 33]. The von Karman length scale $\left(L_{\mathrm{vk}}\right)$ composed of the first and second velocity derivatives is not sensitive to grid efforts but dynamically adjusts the resolution to the spectra content. Thus, it is an appealing element that has a strong theoretical foundation and enables flow mode to smoothly vary from RANS to LES-like mode. Moreover, when in the stable region, $L_{k \omega}<L_{\mathrm{vk}}$ and $Q_{\mathrm{SAS}}=0$, returning a pure RANS mode, while when in the unstable region, $L_{k \omega}>L_{\mathrm{vk}}$ and $Q_{\mathrm{SAS}}>0$ and the mode will turn to LES-like mode.

2.4. ISAS Model Formulation. To combine the DES and SAS concepts based on the SST model, a simple way is to replace the grid scale in (6) with the von Karman scale.
As known, using Reynolds averaging method, the standard two-equation models such as SST always produce a turbulent length scale $(L)$ proportional to the thickness of the boundary layer $(\delta)$, which is suitable for the stable shear flows near walls but depresses the resolved scales in the unsteady regions. When using the hybrid methods such as DES and SAS, the relation between $L$ and $\delta$ in the RANS regions is still [20]

$$
L \sim \delta,
$$

whereas in the separated regions, the grid scale $\Delta$ and the von Karman scale $L_{\mathrm{vk}}$, respectively, used in DES and SAS allow the solution to adapt to the resolved structures in a classical LES-like behavior and gradually reach to convergent solutions. The current relations between different length scales can be expressed as

$$
\begin{array}{r}
\text { DES : }\left(l_{k \omega} \sim C_{1} \Delta\right) \ll \delta, \\
\text { SAS : }\left(L_{k \omega} \sim C_{2} L_{\mathrm{vk}}\right) \ll \delta,
\end{array}
$$

where the model constants $C_{1}$ and $C_{2}$ are of the order of one. Moreover, considering the equilibrium assumptions (balance between production and destruction of the turbulence energy), the eddy viscosity of each method is of the following relation $[21,26]$ :

$$
\begin{aligned}
v_{t}^{\mathrm{DES}} & =\left(\beta^{* 3 / 4} C_{\mathrm{DES}} \cdot \Delta\right)^{2} S, \\
v_{t}^{\mathrm{SAS}} & =\left(\sqrt{\left.\left(\frac{\beta}{C_{\mu}}-\alpha\right) /\left(\kappa \xi_{2}\right) \cdot L_{\mathrm{vk}}\right)^{2} S .}\right.
\end{aligned}
$$

It is shown that both the two formulations have the similar structure as the LES viscosity:

$$
v_{t}^{\mathrm{LES}}=\left(C_{s} \cdot \Delta\right)^{2} \cdot S,
$$

where the $C_{s}$ value is also of the order of one and thus the $\Delta$ and $L_{\mathrm{vk}}$ scales are well compatible with each other and it is theoretically acceptable to the replacement.

Through the brief derivations above, the new proposed hybrid function employs von Karman scale instead of grid scale in $(6)$, and the dominant factor $\left(F_{\text {ISAS }}\right)$ of which is reformulated as

$$
F_{\text {ISAS }}=\max \left(\frac{l_{k \omega}}{C_{\text {ISAS }} L_{\mathrm{vk}}}, 1.0\right),
$$

where $C_{\text {ISAS }}$ is the control parameter with the value of 1.67 calibrated optimal in the next section.

With the modification in (13), the new model can effectively adjust the turbulent fluctuations by directly operating on the $k$ equation like DES, and more importantly, the $L_{\mathrm{vk}}$ scale enables the model to dynamically provide the eddy viscosity with the concept of scale-adaptive; thus, the underlying method is named improved scale-adaptive simulation (ISAS).

2.5. ISAS Model Calibration. In (10), the DES constants $\beta^{*}=0.09, \quad C_{\mathrm{DES}}=0.61$. In $(11)$, the SAS parameter 
$\sqrt{\left(\left(\beta / C_{\mu}\right)-\alpha\right) /\left(\kappa \xi_{2}\right)}$ appears in [21] associated with high wave number damping. We just calibrate the whole part $\sqrt{\left(\left(\beta / C_{\mu}\right)-\alpha\right) /\left(\kappa \xi_{2}\right)}$ in the DHIT case like that done in [34]. Finally, we found 0.42 is feasible for the proportional relation. From the above derivation, we get the scale relation in the following:

$$
\begin{aligned}
v_{t}^{\mathrm{DES}} & =\left(\beta^{* 3 / 4} C_{\mathrm{DES}} \cdot \Delta\right)^{2} S \approx(0.1 \Delta)^{2} S \\
\nu_{t}^{\mathrm{SAS}} & =\left(\sqrt{\left.\left(\frac{\beta}{C_{\mu}}-\alpha\right) /(\kappa \xi) \cdot L_{\mathrm{vk}}\right)^{2} S \approx\left(0.42 L_{\mathrm{vk}}\right)^{2} S .}\right.
\end{aligned}
$$

Assuming that SAS and DES models produce the same energy dissipation, we can obtain $(0.1 \Delta)^{2} S=\left(0.42 L_{\mathrm{vk}}\right)^{2} S$ and $\Delta=4.2 L_{\mathrm{vk}}$. If simply replace the grid scale in (6) with von Karman scale and avoid excessive dissipation, an approximate range $C_{\mathrm{ISAS}} \leq C_{\mathrm{DES}} * 4.2=2.56$ will be conducted. Meanwhile, one similar relation based on (11) and (12) has been built in [21] with an optimal $C_{s}=0.11$. We assume the SAS method matches the same energy spectrum of LES and the equation $\Delta=3.82 L_{\mathrm{vk}}$ can be gained. Again, roughly use $L_{\mathrm{vk}}$ instead of $\Delta$ in (6) and avoid more dissipative action, and we can get $C_{\mathrm{ISAS}} \leq C_{\mathrm{DES}} * 3.82=2.33$. It is very close between the two rough results.

To obtain the optimal value of $C_{\text {ISAS }}$, a recommended way is to calculate the simple decaying homogeneous isotropic turbulence (DHIT), for example, the classical test case performed by Comte-Bellot and Corrsin [35], just as that did for most LES and hybrid models calibrations [19, 29, 32, 36]. The experimental equipment was simplified to a cubical box with periodic boundary conditions for the ISAS simulations, and a uniform grid with $32 \times 32 \times 32$ points was used. Moreover, the numerical strategies such as the initial conditions and splitting schemes are drawn from the similar DES calibrations $[29,32]$. Figure 1 shows the spectra of the resolved kinetic energy for the selected DHIT case at the normalized time $t=2$; meanwhile, the ISAS outputs with different $C_{\text {ISAS }}$ and the line with $-5 / 3$ slope are also given for comparison. As displayed, the energy spectra change as using different $C_{\text {ISAS }}$ values; concretely, the lower the value, the less the dissipation. However, the curve with $C_{\mathrm{ISAS}}=1.67$ best matches the measurement and provides the spectral slope close to the $-5 / 3$ near the cutoff wave number, and the declared $C_{\text {ISAS }}$ value fairly fits the rough estimate above.

Through the simple validation in the freely decaying isotropic turbulence, it turns out that, with a proper constant, the ISAS model allows for the formulation of the turbulence spectrum, which is contrary to URANS but similar to DES and SAS. As a result, the $C_{\text {ISAS }}$ value 1.67 is proven optimal, and more comprehensive validation will be presented in the next section.

\section{Generic Validation of ISAS Model}

Flow over a circular cylinder is a typical case of bluff body flows, which is often involving complex vortex motion and

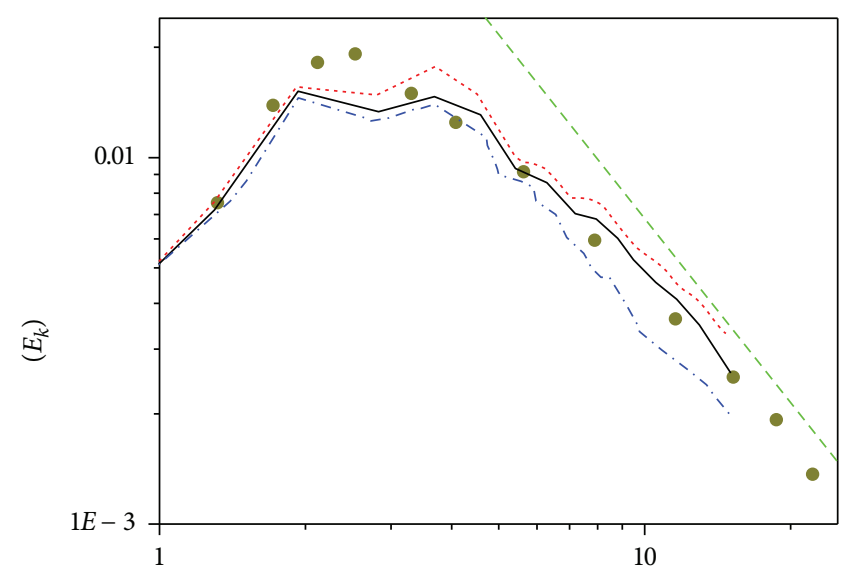

$(k)$

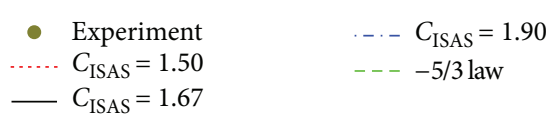

FIGURE 1: Comparison of energy spectra between ISAS simulations and DHIT data.

rich physics such as separation and reattachment. Because of the simple shape and representative flow phenomena, the cylinder flow is often favorable for the validation of turbulence models. As mentioned above, the flow over a circular cylinder at $\mathrm{Re}=3900$ (corresponding to cylinder diameter and freestream velocity) is selected for the thorough validation of the proposed ISAS model, mainly due to the large amount of experimental and numerical data in this particular subcritical Reynolds number.

\subsection{Numerical Solution}

3.1.1. Grid Generation and Boundary Conditions. All threedimensional simulations were performed on a multiblock grid extruding from the cross-sectional plane, displayed in Figure 2(a), which is overall of hexahedral volumes, and an O-type mesh is designed near walls to keep better orthogonality. With the goal of discussing the grid-independent ability of the adopted models, it should be noted that two grids were, respectively, generated, which differ from each other in the expansion ratios for the RANS region and wake region. Specifically, as shown in Figure 2(b), a growth rate of 1.04 is used in RANS region for the relatively fine grid while a rate of 1.07 is used for the coarse one, other than that, the grid spacing of $0.15 D$ and $0.2 D$ is, respectively, used for the two grids in the wake region. As a result, a total of $225 \times 158$ nodes were distributed in the $x-y$ plane of the fine grid; meanwhile, $166 \times 126$ nodes were adapted to the coarse grid. Finally, to confirm the $y^{+}$values well below 1.0 at the wall, the minimum wall grid spacing is set to $2 \times 10^{-3} \mathrm{D}$ [37], and the growth rate of both grids is below 1.1 to keep enough nodes in boundary layer.

Concerning the boundary conditions, to prevent the undesirable effort from inflow and outflow, a fixed velocity field $(0.05 \mathrm{Ma})$ was used at inflow plane and a distance of $20 D$ was developed from the cylinder wall, while a 


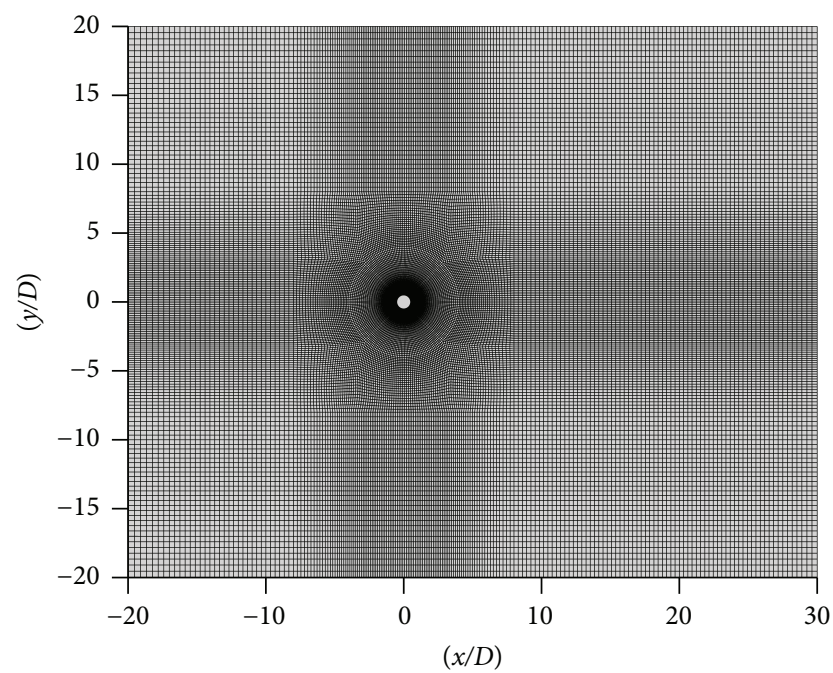

(a) $x-y$ plane

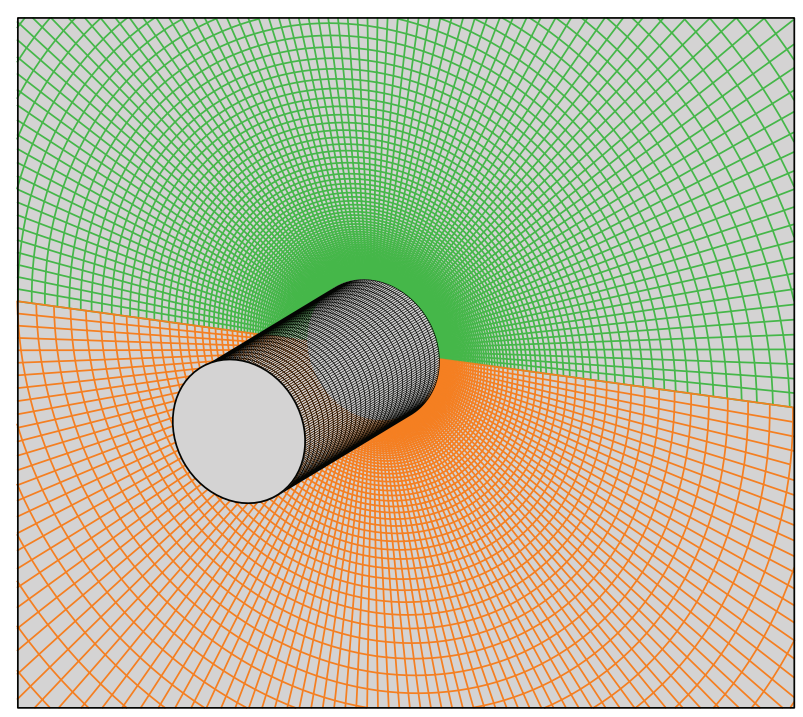

(b) Zoom of the near-wall region

Figure 2: The design of cross-sectional grid (a) overview and (b) near-wall region (top, fine grid; bottom, coarse grid).

nonreflecting condition with the $30 \mathrm{D}$ distance was set for the outflow plane. The inviscid wall condition was applied to both the top and bottom sides, while the space between each side and cylinder wall is also $20 \mathrm{D}$. As for the spanwise direction with a periodic assumption, the proper $\pi D$ extent and normal 50 nodes were adopted for both fine and coarse grids aiming at fully reproducing the threedimensionality [38-40].

3.1.2. Numerical Strategies. All the solutions presented in this paper are based on a 3-D incompressible solver. The space discretization of the governing equations was performed using the popular second-order finite volume method (FVM), and a second-order implicit backward method was used for time integration coupled with the dynamically adjustable stepping technique [41], while the transient PISO algorithm was employed for the pressure-velocity coupling. Concerning the convective terms which mostly affect the solving precision, both the velocity and turbulent terms were approximated using a high-resolution NVD Gamma scheme [42]. Finally, the linear systems were solved with the GaussSeidel method. It is worth mentioning that, to achieve stable and convergent calculations for every time step, a local Courant number close to 0.5 was adopted as well as an accuracy of $10^{-6}$ for pressure and $10^{-7}$ for other dependent variables.

It is well known that periodical vortex shedding exists in the cylinder flow at current Re, and sufficiently long physical time integration is very essential for the accurate predictions of the average surface forces. Therefore, in order to get reliable statistics, a total time of about 60 shedding cycles $\left(T=300 D / U_{\infty}\right)$ was taken for each simulation, and the final time-spatial averaged data were recorded over approximate 50 cycles with the initial 10 cycles ignored. The focused time is more than 40 shedding cycles suggested by Franke and Frank [43].

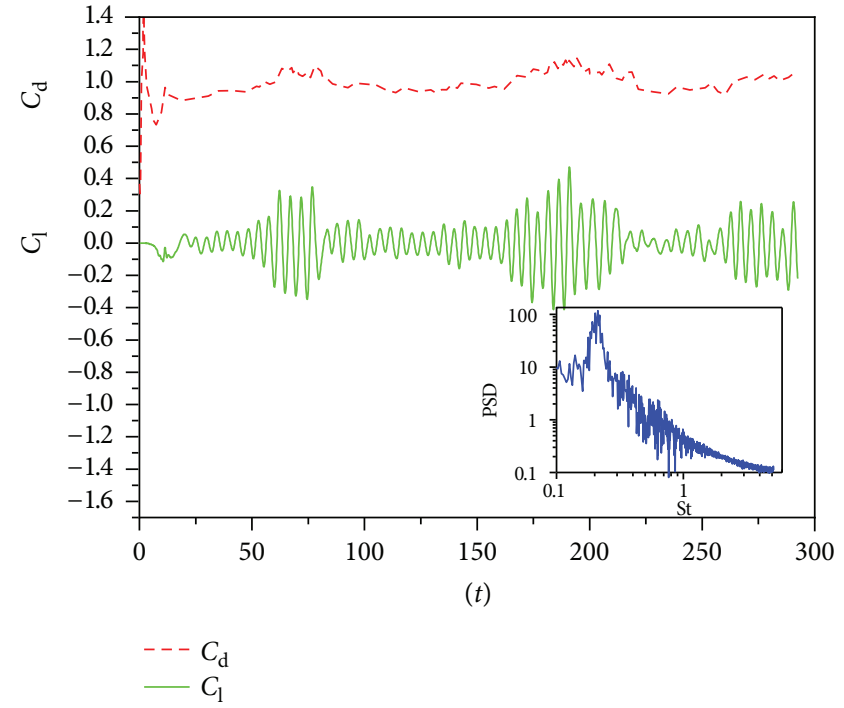

FIgURE 3: Time history of force coefficients using ISAS model and fine grid (lift, $C_{\mathrm{l}}$; drag, $C_{\mathrm{d}}$ ).

Figure 3 shows the time history of $\operatorname{drag}\left(C_{\mathrm{d}}\right)$ and lift $\left(C_{\mathrm{l}}\right)$ coefficients for the ISAS simulation performed on the fine grid. As shown, the lift amplitude is unstable but appears periodical wave, while the drag magnitude appears strong fluctuation near an average value. Moreover, the Strouhal number (St) corresponding to shedding frequency is also given using a fast Fourier transform (FFT) to the lift signal with time. In addition, the mean force coefficients as well as Strouhal numbers of all simulations are listed in Table 1.

3.2. Results and Discussions. In this section, all numerical results with available experimental data are presented and detailed comparisons are conducted between ISAS, 
TABLE 1: Summary of the statistical flow quantities for the flow over a cylinder flow at $\mathrm{Re}=3900$.

\begin{tabular}{|c|c|c|c|c|c|c|}
\hline Case & $C_{\mathrm{d}}$ & $L_{\mathrm{r}} / D$ & $-U_{\min } / U_{\infty}$ & $-C_{\mathrm{pb}}$ & St & $\theta_{\text {sep }}$ \\
\hline Exp. [44] & - & 1.51 & 0.34 & - & 0.21 & - \\
\hline Exp. [46] & 0.98 & - & - & 0.94 & - & - \\
\hline ISAS1 & 1.00 & 1.496 & 0.296 & 0.915 & 0.214 & 87.24 \\
\hline DES1 & 0.95 & 1.857 & 0.308 & 0.878 & 0.207 & 86.95 \\
\hline SAS1 & 1.01 & 1.485 & 0.285 & 0.964 & 0.212 & 87.90 \\
\hline ISAS2 & 1.03 & 1.348 & 0.264 & 1.002 & 0.208 & 88.10 \\
\hline DES2 & 1.06 & 1.271 & 0.287 & 1.037 & 0.203 & 88.47 \\
\hline SAS2 & 1.06 & 1.213 & 0.294 & 1.044 & 0.205 & 88.68 \\
\hline DNS [48] & - & 1.41 & 0.291 & - & 0.20 & - \\
\hline LES [38] & 1.04 & 1.35 & 0.37 & 0.94 & 0.21 & 88 \\
\hline LES-SMAG [41] & 1.18 & 0.9 & 0.26 & 0.8 & 0.19 & 89 \\
\hline LES-THE [41] & 0.97 & 1.67 & 0.27 & 0.91 & 0.21 & 88 \\
\hline SST-DES [37] & 1.01 & 1.46 & 0.29 & 0.89 & 0.20 & 86.4 \\
\hline SST-PANS [37] & 1.06 & 1.20 & 0.28 & 0.96 & 0.20 & 87.1 \\
\hline SA-DES, [40] & 1.20 & 0.85 & 0.3 & 1.08 & 0.22 & 89.3 \\
\hline SA-DDES [49] & 0.97 & 1.37 & 0.28 & 0.97 & 0.22 & 88.3 \\
\hline SA-IDDES [40] & 1.02 & 1.42 & 0.28 & 0.89 & 0.22 & 87 \\
\hline$V^{2}-f$-DES $[40]$ & 0.99 & 1.68 & 0.4 & 0.83 & 0.21 & 86.4 \\
\hline
\end{tabular}

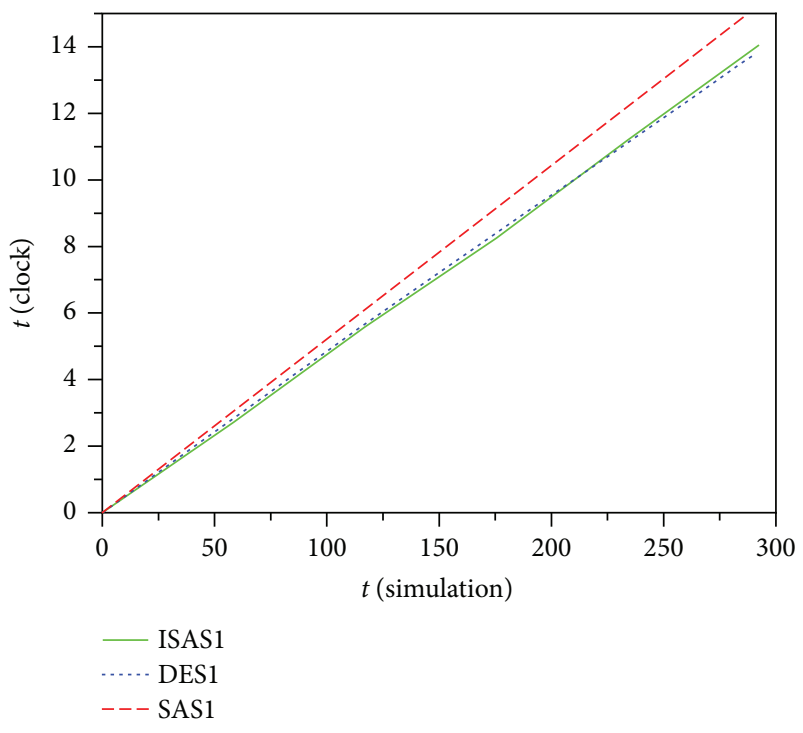

(a) Fine grid

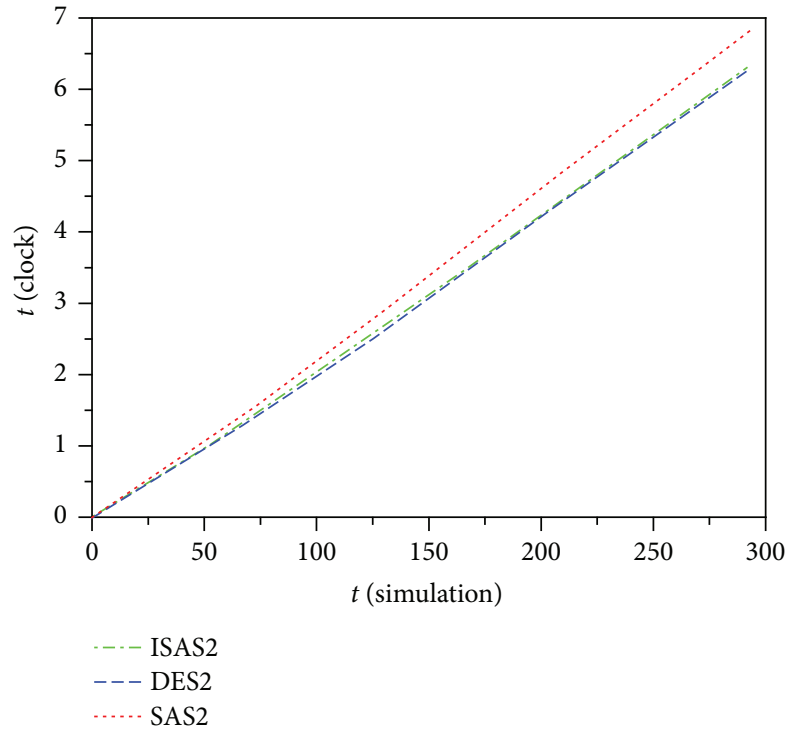

(b) Coarse grid

Figure 4: Computational efficiency of ISAS, DES, and SAS.

DES, and SAS models. It is worth mentioning that the following terminologies will be used for the subsequent simulations with the goal of briefly and avoiding confusion: the results based on the fine grid for the three models are, respectively, named ISAS1, DES1, and SAS1 while those based on the coarse grid are named ISAS2, DES2, and SAS2.

3.2.1. Comparison in the Computational Efficiency. To compare the efficiency of different models, all simulations were performed on the same workstation with 40 cores. It should be emphasized that all the simulations used the same numerical setup and the iteration in every time step was ensured convergent; thus, the model efficiency just depends on the solving speed of the turbulent equations.

The computational efficiency of ISAS, DES, and SAS methods in both fine and coarse grids is discussed in Figure 4, in which the longitudinal axis is the flow time normalized with a ratio of $U_{\infty} / D$ while the transverse axis is the local clock time normalized with one hour (h). As shown, the six relations between flow time and clock time appear to be oblique lines and, respectively, with different 


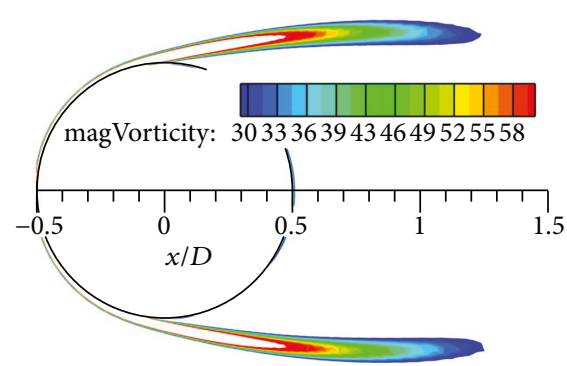

(a) ISAS1

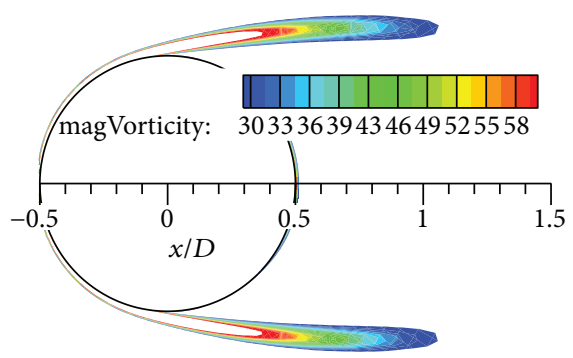

(d) ISAS2

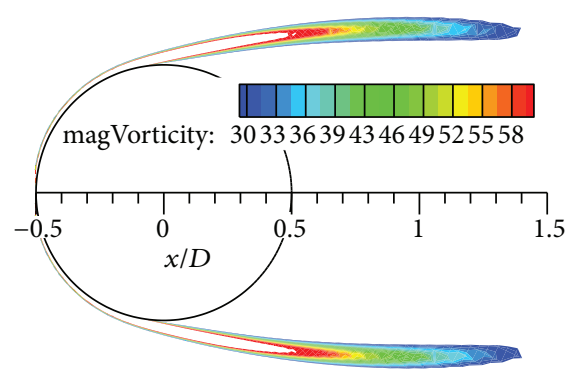

(b) DES1

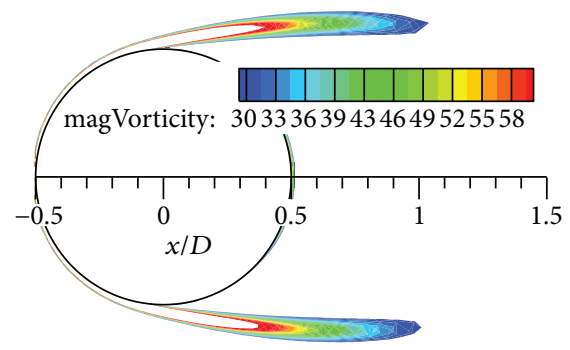

(e) DES2

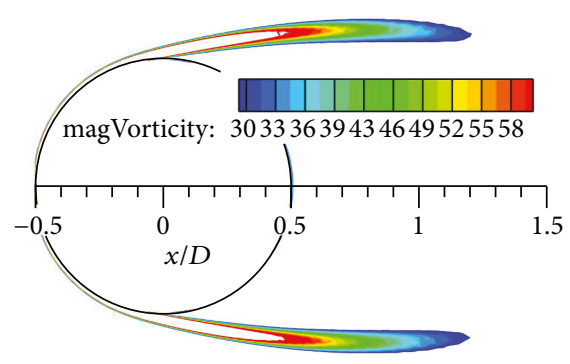

(c) SAS1

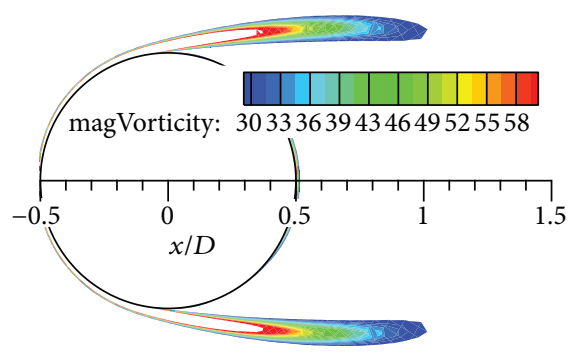

(f) SAS2

FIGURE 5: Separating shear layers displayed by the mean vorticity magnitude.

slopes. Moreover, the efficiency of ISAS appears very close to DES but drastically superior to SAS, and this trend is more obvious over flow time, which is reasonable due to different model formulations. The work mechanism using the additional source term $Q_{\text {SAS }}$ in SAS is more complex than that only using the ratio of turbulent length scale to the von Karman length scale in ISAS, which leads the SAS equations more expensive to be solved, while with a similar formulation, there is a tiny gap between ISAS and DES efficiency though the $L_{\mathrm{vk}}$ scale is somewhat more complicated than grid scale.

3.2.2. Flow Statistics. It is well known that the thin shear layers with about one cylinder diameter length are the distinct feature of the cylinder flow at current $\mathrm{Re}=3900$ [38]. As observed in Figure 5, all the simulated shear layers are displayed by the mean vorticity magnitude. Both on the fine and coarse grids, all the three comparative models well represent the development of the laminar shear layers originated from the solid surface, which is especially benefited from the acceptable capacity of SST model in calculating the attached boundary layer. However, through a further inspection, the length relations among the results are DES1 > ISAS1 > SAS1, meanwhile, ISAS2 $>$ DES2 $>$ SAS2, mainly due to the different grid sensitivity and adjusting mechanism. The shear layers from fine grid are longer than those from coarse grid while the DES1 result from the fine grid is noticeably longest. From Figure 5, we can visibly observe the diverse model performances in simulating the shear layers but the exact length of shear layers still needs quantitative validation.

Figures 6 and 7 depict the time and space averaged velocity data extracted from different simulations; the available experimental data at same Reynolds number are listed below:

(1) PIV (particle image velocimetry) data measured by Parnaudeau et al. [44]
(2) HWA (hot-wire anemometry) data measured by Ong and Wallace [45]

Figure 6 shows the mean streamwise velocity along the wake centerline. As shown for clarity in Figure 6(a), the DES1 profiles from fine grid appear a terribly delayed development of the recirculation region by comparison with measurements, and the direct features for which are reflected on a larger recirculation length $\left(L_{\mathrm{r}} / D\right.$, the distance between the cylinder surface and the peak with 0 streamwise velocity) and a slower velocity recovery after the recirculation region. Nevertheless, the accurate recirculation size and fastest velocity recovery of ISAS1 give the best agreement with the experimental data. Finally, the SAS1 results give a similar recirculation length as ISAS1 but with larger discrepancy in the velocity peak and recovery. The similar results can be observed in Figure 7 which demonstrates the concrete velocity profiles in the near wake region. Concerning the DES1 results on the fine grid (Figures 7 (a) and $7(\mathrm{~b})$ ), it shows a delayed development of the mean streamwise velocity from the $\mathrm{U}$ shape to $\mathrm{V}$ shape and the transverse velocity from antisymmetrical shape to declining shape. Meanwhile, the ISAS1 result gives a better recirculation size and a faster velocity recovery than the SAS1 result. When using the coarse grid, all the simulated accuracy is reasonably reduced as shown in Figures 6(b), 7(c) and 7(d). The accuracy of ISAS, DES, and SAS models is nearly at the same level compared to the measurements, but the ISAS2 results are concretely more accurate than those of the other two methods, though underestimate the velocity peak in Figure 6(b).

Combining the analysis of Figures 5-7, the fact is revealed that the reproduction of the recirculation region is specifically associated with the development of the shear layers. Thus, the recirculation data such as region size and velocity profiles are applicable to validate the accuracy of the shear layers. More concretely, with extremely longer 


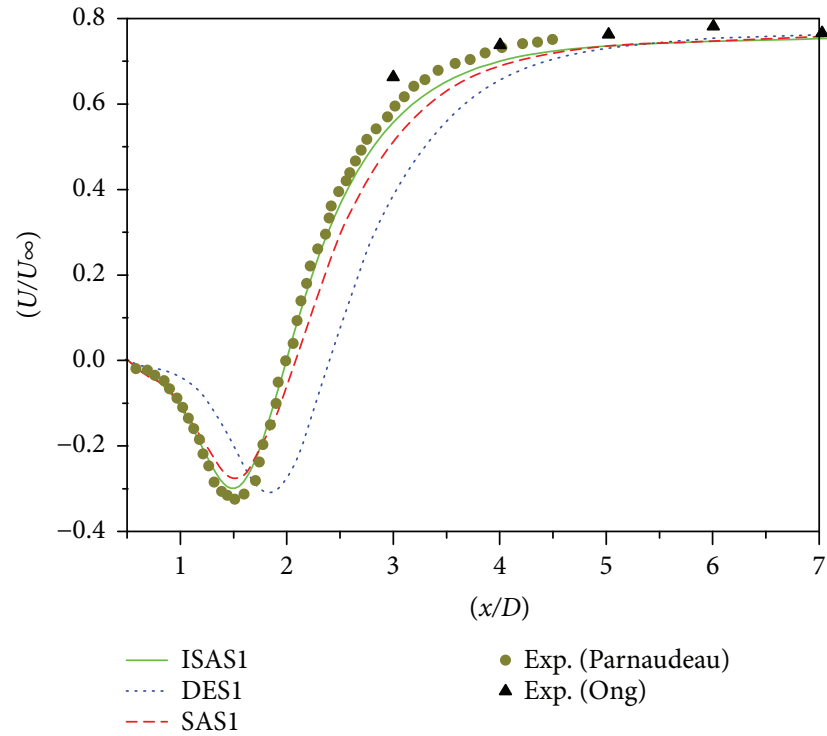

(a) Fine grid

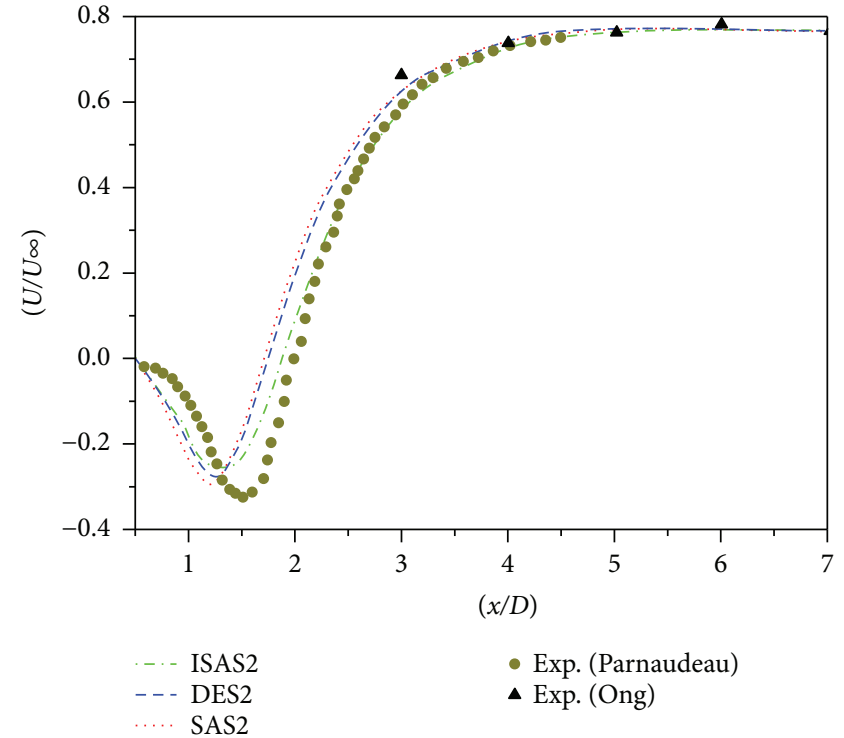

(b) Coarse grid

FIGURE 6: Distribution of mean streamwise velocity along the wake centerline.

length of shear layers, the DES model appears a delayed development of the recirculation region on fine grid, mainly due to the inherent grid-dependence feature. With the concept of scale-adaptive, both ISAS and SAS results do not appear exaggerated discrepancies considering different grids, and with a more accurate simulation of the shear layers, the recirculation profiles of ISAS simulation are accordingly closer to measurements. In addition, the specific results of the recirculation length $\left(L_{\mathrm{r}} / D\right)$ and the peak of back flow velocity $\left(U_{\min } / U_{\infty}\right)$ are listed in Table 1.

From the above, we have simply discussed the relation between the simulation of boundary layer and grid using different turbulent models, while the ability of ISAS to simulate the separated flows is primitively validated. The further explanations for the LES-like resolution are based on the theoretical formulation and the physical mechanism of the three models. It is well known that the hybrid methods adjust the viscosity to different levels regarding the near-wall region and separated region in different ways. Considering the proposed ISAS model in this paper, it has a theoretical mechanism similar to DES but a physical mechanism like SAS.

Noting the different scales on the colour bar, the instantaneous ratio of the modelled turbulent length scale to the dominant length scale for different models is displayed in Figure 8. With a similar limiter formulation in the first $k$-scale equation as DES, the switch of the ISAS resolution from RANS to LES region is achieved by a comparison of the RANS scale $(L)$ with the local von Karman scale $\left(L_{\mathrm{vk}}\right)$ rather than the grid scale used in DES. Both the $L_{\mathrm{vk}}$ and $\Delta$ scales are passive in the boundary layer and active in the detached region, which is distinguished by the dividing factor $L / L_{\mathrm{vk}}=1.0$ or $L / \Delta=1.0$. Thus, as shown in Figures $8(\mathrm{a}), 8(\mathrm{~b}), 8(\mathrm{~d})$ and $8(\mathrm{e})$, the dominant turbulent length scales of ISAS and DES are nearly in the same level but visually differ from each other in the adjusting effort. For DES on fine grid, the grid scale is undesirably earlier activated and the switch from RANS to DES takes place somewhere inside the boundary layer, which will lead to the gray area issue and introduce uncertainty and inconsistent solution to the primary vertex, whereas this issue can be overcame in ISAS benefited from the $L_{\mathrm{vk}}$ scale, which is determined by the local flow physics and provides a finer adjusting effort for the flow unsteadiness. It should also be mentioned that the $F_{\text {ISAS }}$ scale distribution (using fine mesh) fits the mechanism of hybrid approach very well with no very large value occurred, as plotted in Figure 9.

Both treating $L_{\mathrm{vk}}$ as the defining scale, the adjusting effort of ISAS is visually similar to SAS as shown in Figures $8(\mathrm{a})$, $8(\mathrm{c}), 8(\mathrm{~d})$ and $8(\mathrm{f})$. Both of them are independent to the grid spacing near wall and automatically adapt the resolvable flow scales down to the grid limit in separated region. Nevertheless, the adjusting mechanism of ISAS is not identical with SAS, and the main distinction between each other lies in the different adjusting formulation. In SAS, the switch from RANS to SAS mode is controlled by the additional source term $\left(Q_{\mathrm{SAS}}\right)$ in the second $\omega$-scale equation, which therefore adjusts the flow by the change of the dissipation rate, and the dividing point from RANS to LES-like is near $L / L_{\mathrm{vk}}=0$. By contrast, the ISAS adjusting formulation directly acting on the dissipative term of $k$ equation is more efficient.

The mean turbulent viscosity $\left(\mu_{t} / \mu\right)$ is presented in Figure 10. The viscosity level of ISAS is more consistent with that of DES results and lower than that of SAS on both the fine and coarse grids, which depicts that the SAS model is maybe more dissipative based on current grids. Meanwhile, concerning the DES results, it appears an extremely lower area near wall when using fine grid, which means the RANS mode is prematurely destroyed but the local grids are not dense enough for the true LES simulation, which is consistent with the analysis of the DES mechanism.

As the additional information to the first-order velocity magnitude, the time and space averaged Reynolds stresses 


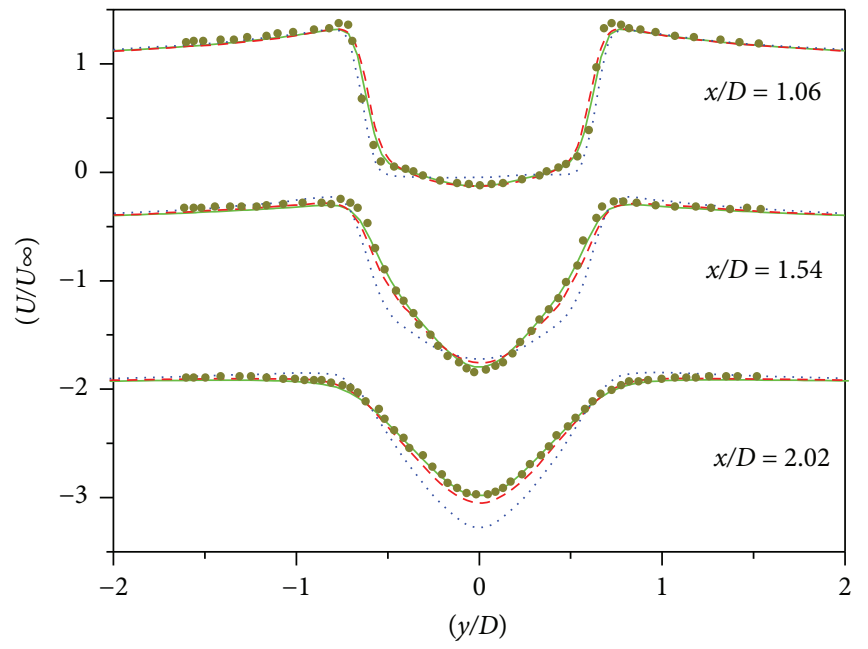

(a) Streamwise velocity (fine grid)

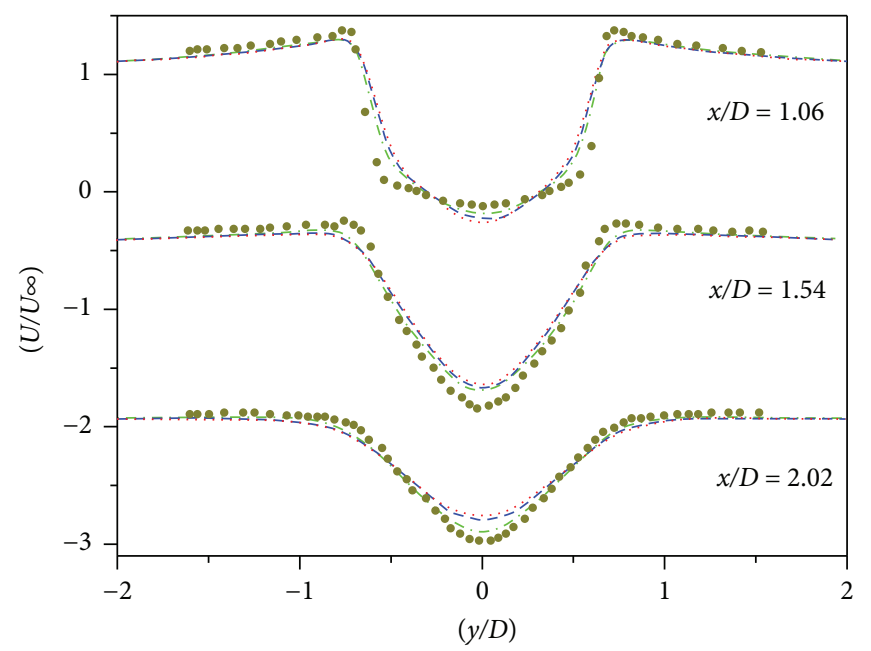

(c) Streamwise velocity (coarse grid)

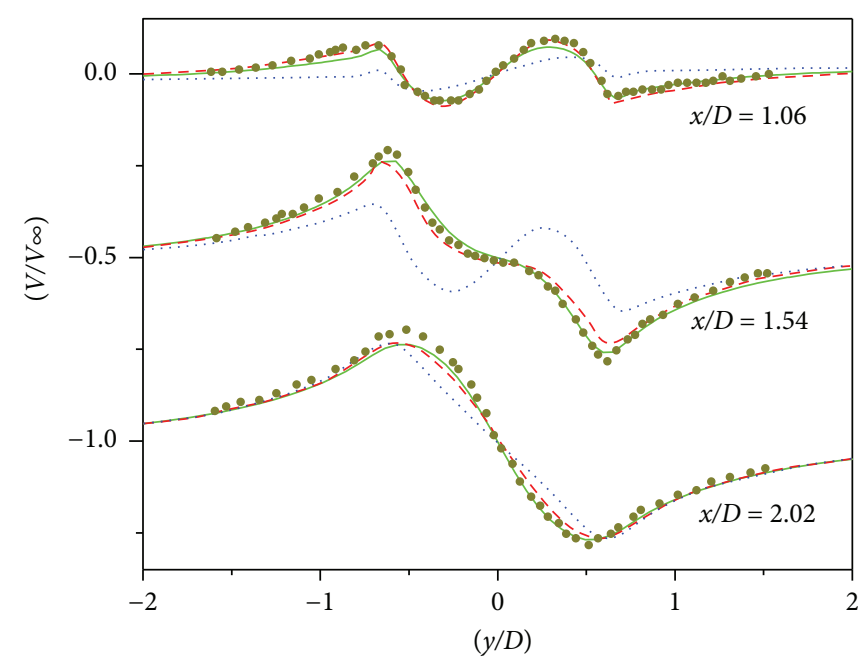

(b) Transverse velocity (fine grid)

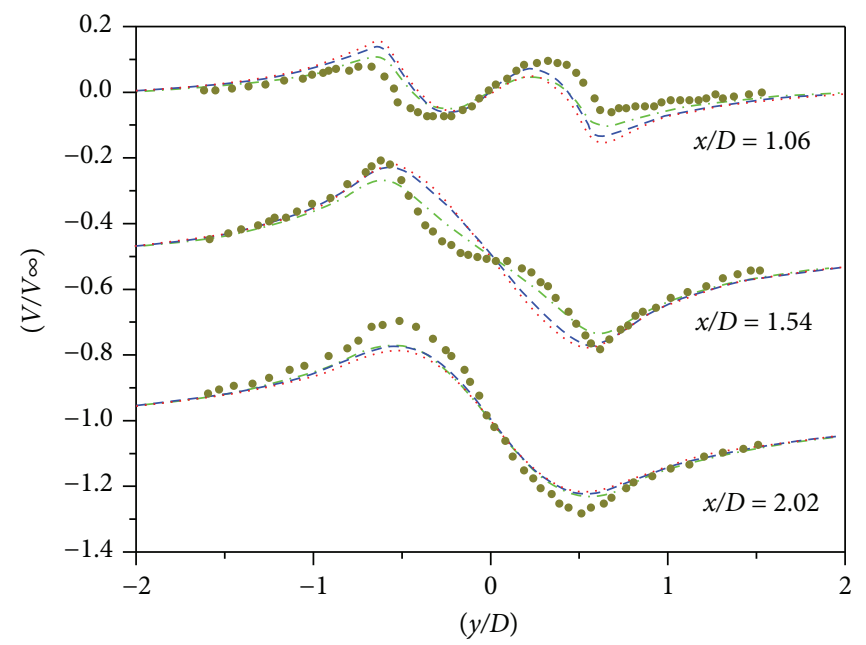

(d) Transverse velocity (coarse grid)

Figure 7: Mean velocity distributions at different sections (for details, see the caption for Figure 6).

are presented in Figure 11. As shown in Figures 11(a) and 11(b), the DES model arises terrible modeled stress depletion (MSD) issue, mainly due to the unacceptable lower viscosity level in the gray area, which leads to a drop of the RANSmodelled stress but without enough LES-resolved stress. At the same time, the MSD phenomenon is not evoked in the ISAS and SAS outputs. However, leaving aside the MSD issue appeared in DES, the agreement with measurements is not perfect for any model especially on the rough grid (Figures 11(c) and 11(d)), and this result is also observed for some other hybrid methods even the LES method, which is maybe associated with the inherent fault of the eddy viscosity models. It is also worth emphasizing that the ISAS result for the Reynolds stress is encouragingly closer to the experimental data than the original DES and SAS models, which is more obvious in near walls $(x / D=1.06,1.54)$, and fairly reproduces the stress peaks in both streamwise and transverse direction.

Figure 12 demonstrates the mean wall parameters composed of the pressure coefficient $\left(C_{p}\right)$ and the vorticity magnitude $(\operatorname{mag}|\Omega|)$. It should be noted that the angle $\theta$ corresponds to the front stagnation point and the reference experimental data in this subsection are listed as follows:

(1) Pressure measurements conducted by Norberg [46], $\operatorname{Re}=4020$

(2) Vorticity measurements conducted by Ma et al. [47], $\operatorname{Re}=5000$

Concerning the mean pressure distribution first, as shown in Figures 11(a) and 11(c), when based on the fine grid, the ISAS result shows the best agreement with the measurement, while the DES and SAS results, respectively, underestimate and overestimate the pressure value, and all the three results overestimate the concerned value based on the coarse grid. Taking the vorticity magnitude into account, although the peak value on both fine and coarse grids is underestimated, all the calculated data are fairly consistent with the measurements, which is especially obvious on the fine grid. It should be mentioned that the simulated discrepancy related to the wall data among ISAS, DES, and SAS is 

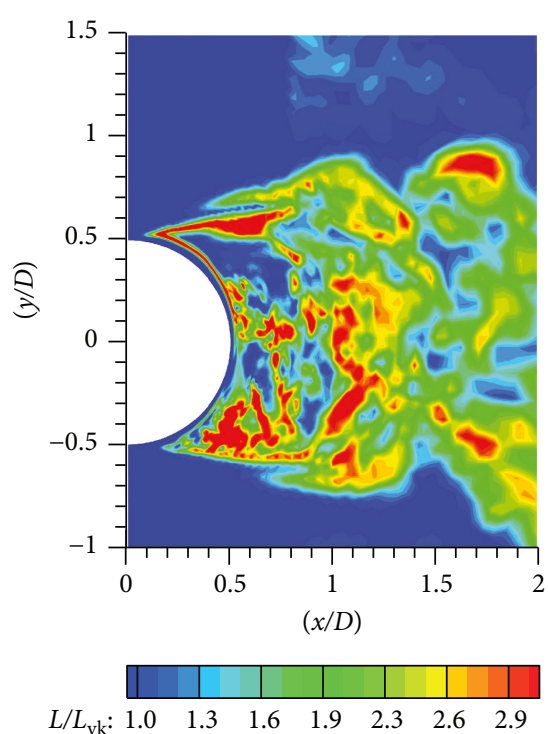

(a) ISAS1
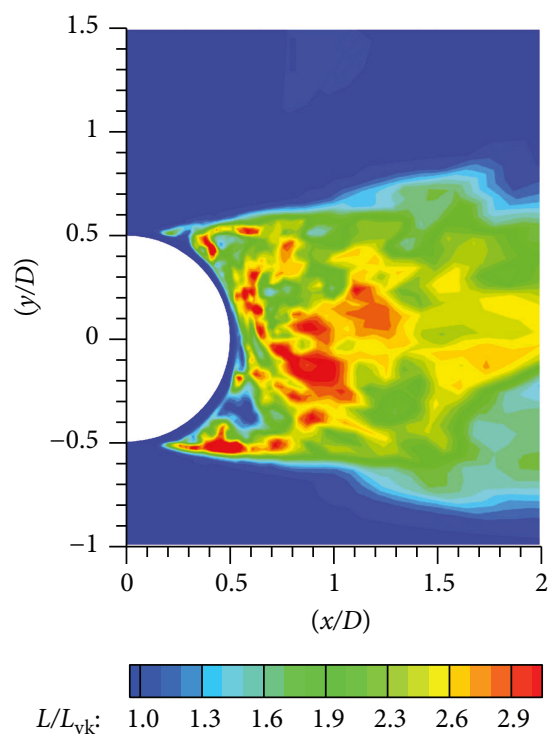

(d) ISAS2

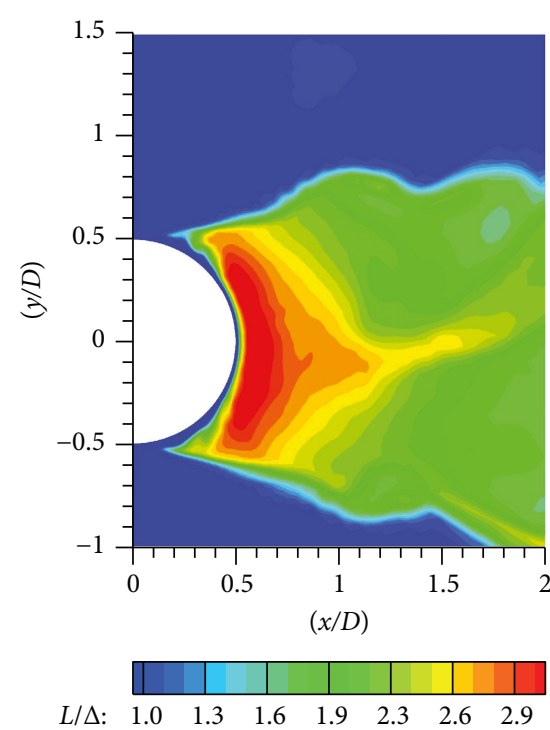

(b) DES1
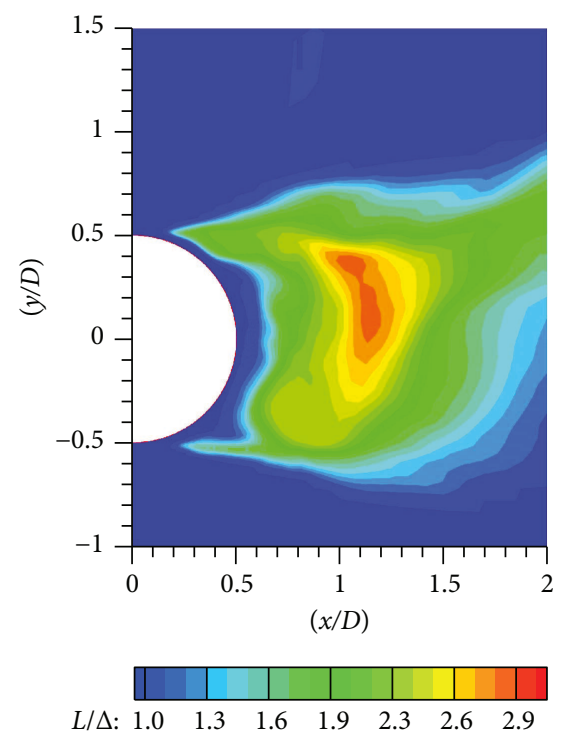

(e) DES2
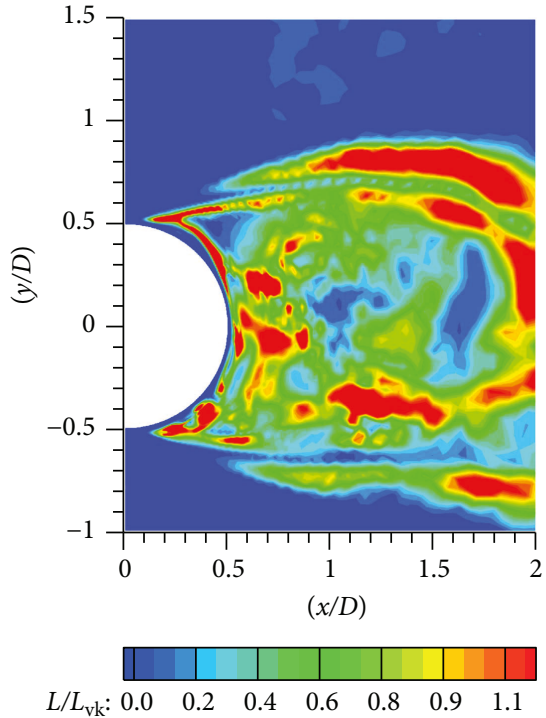

(c) SAS1
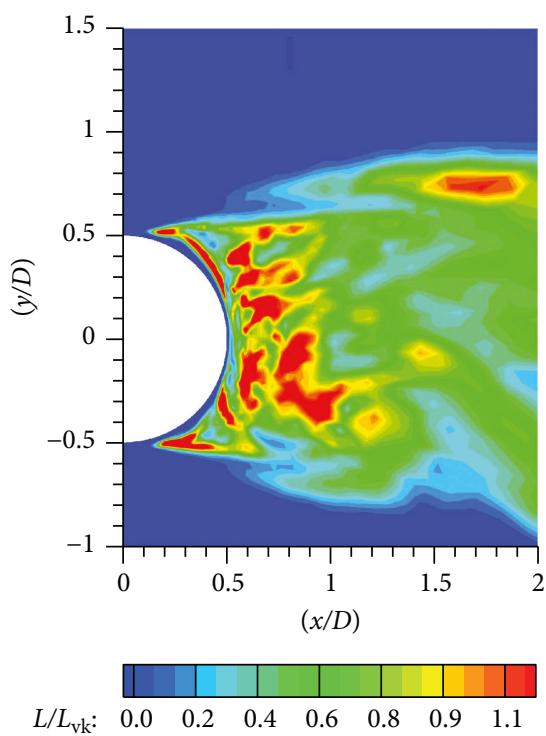

(f) SAS2

FIGURE 8: Contours of the dominant turbulent length scales.
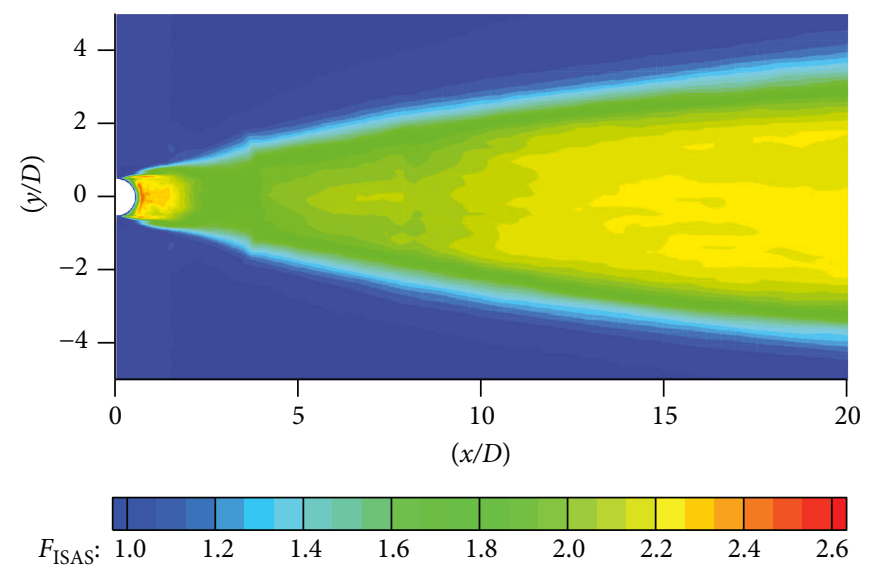

Figure 9: Time-averaged contours of the dominant turbulent length scales (ISAS1). 


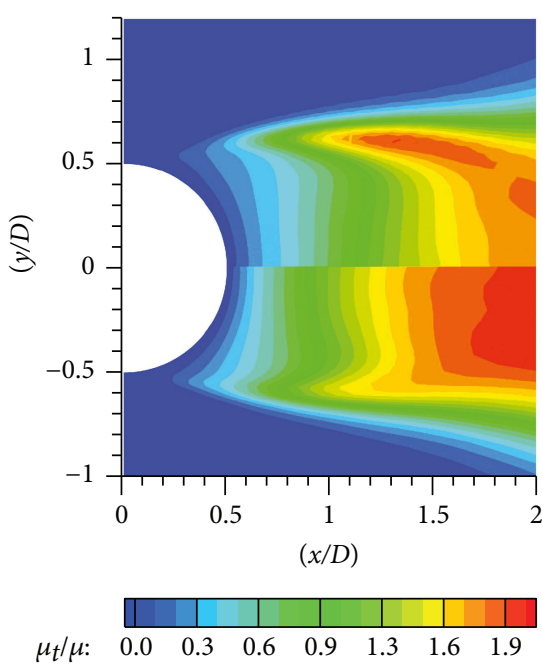

(a) ISAS viscosity

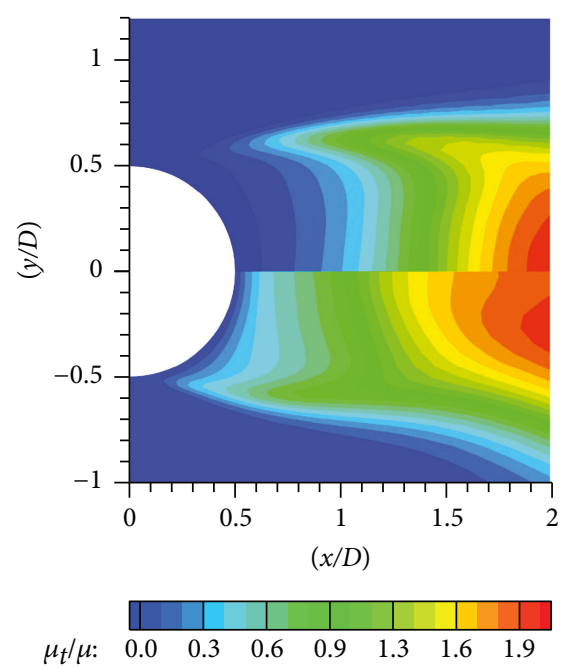

(b) DES viscosity

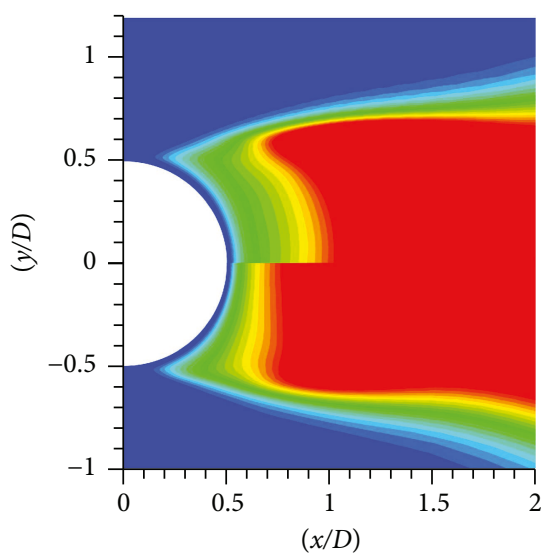

$\mu_{t} / \mu$ :

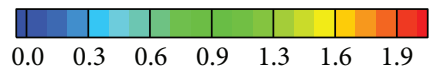

(c) SAS viscosity

FIGURE 10: Contours of mean turbulent viscosity (top, fine grid; bottom, coarse grid).

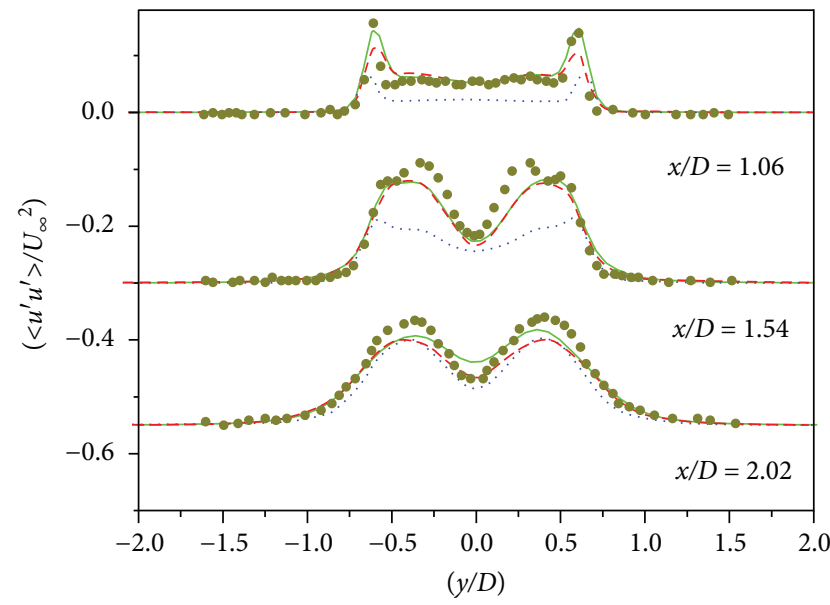

(a) Streamwise Reynolds stress (fine grid)

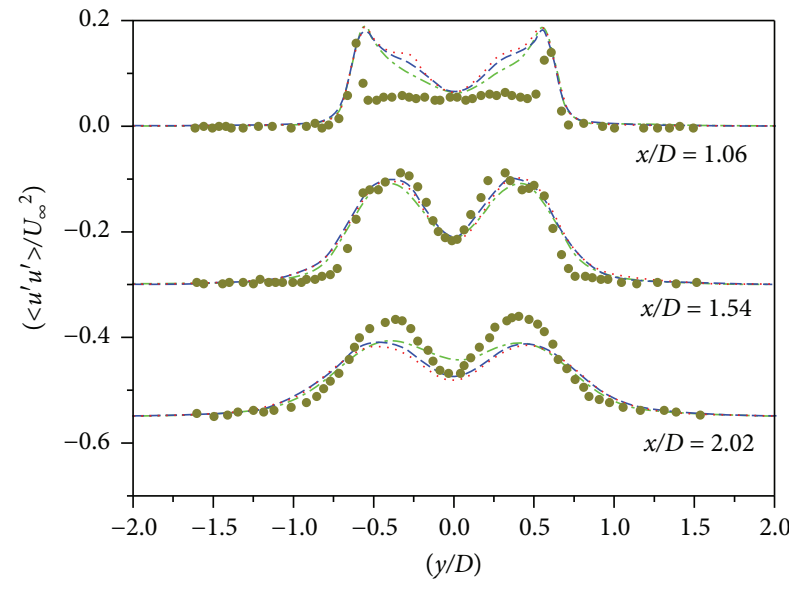

(c) Streamwise Reynolds stress (coarse grid)

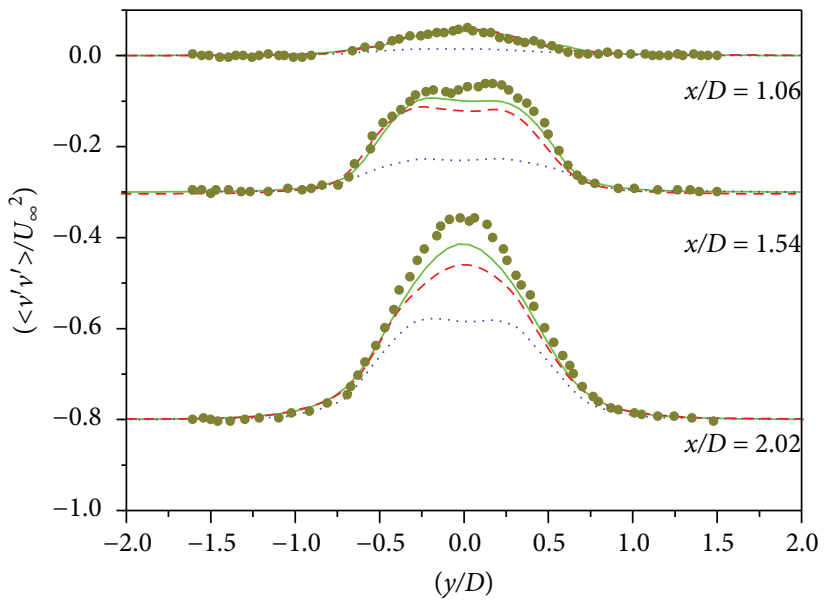

(b) Transverse stress (fine grid)

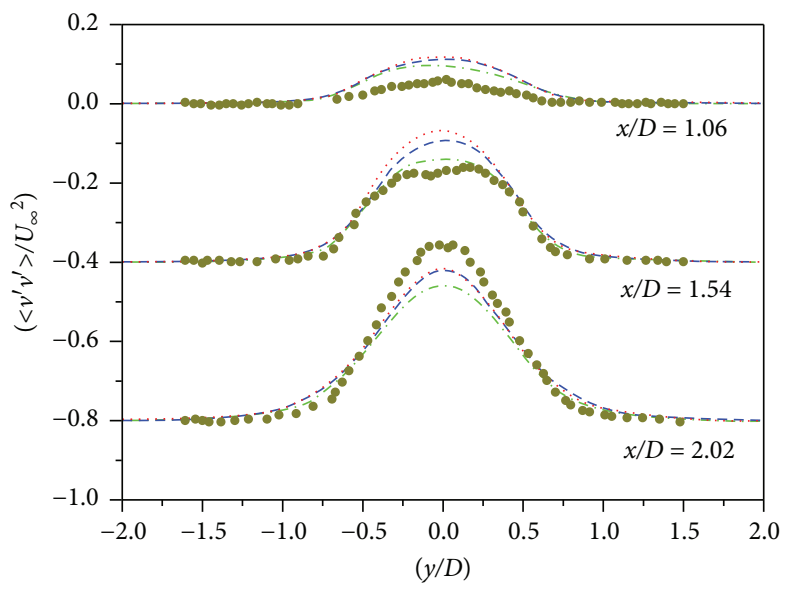

(d) Transverse stress (coarse grid)

Figure 11: Mean Reynolds stress in different sections (for details, see the caption for Figure 6).

very minor and the numerical accuracy is also acceptable compared to measurements. The analysis shows the good ability of the three models in calculating the wall surface, which is greatly benefited from the SST model with strength of accurately and robustly integrating to the wall. Finally, for the sake of comparison, the calculated base pressure 


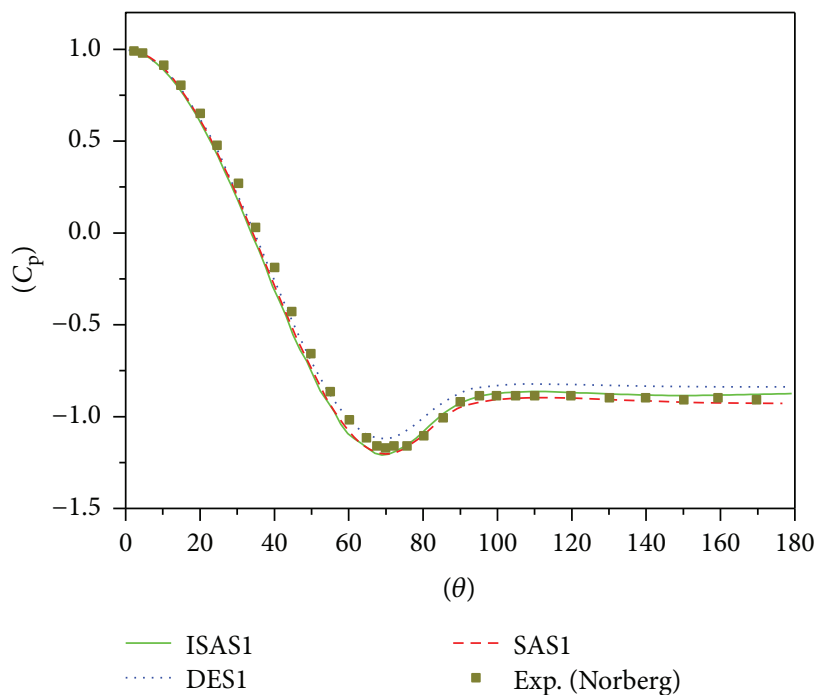

(a) Mean wall pressure coefficient (fine grid)

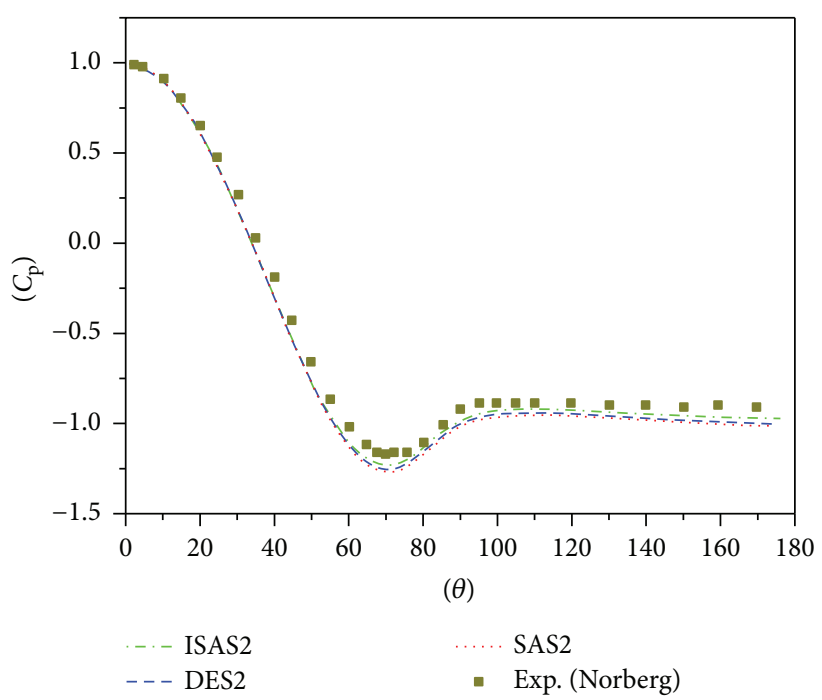

(c) Mean wall pressure coefficient (coarse grid)

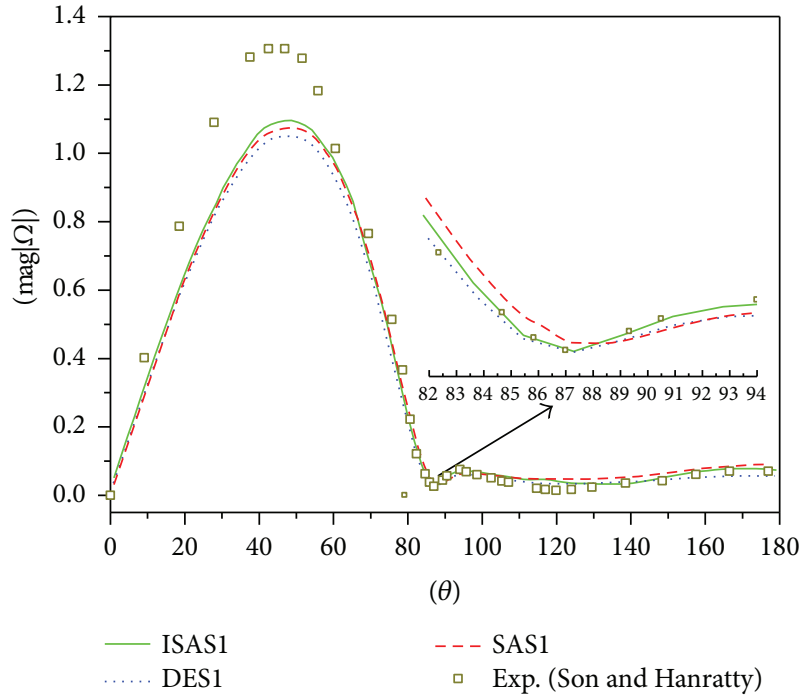

(b) Mean wall vorticity magnitude (fine grid)

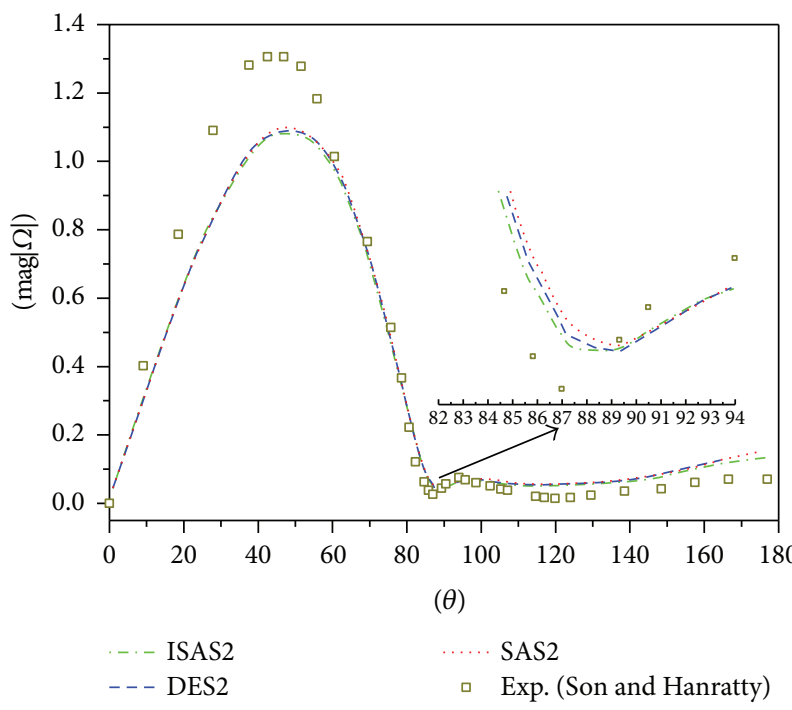

(d) Mean wall vorticity magnitude (coarse grid)

FIGURE 12: Comparison of the mean wall data.

coefficient $\left(C_{\mathrm{pb}}, C_{\mathrm{p}}\right.$ at $\left.\theta=180^{\circ}\right)$ and the separation angle $\left(\theta_{\text {sep }}\right)$ of the attached layer are also listed in Table 1.

Several important integral flow statistics are listed in Table 1, and meanwhile, the available experimental data [48] with relevant numerical results [49] are also presented here as reference. Concerning the parameters associated with the development of the shear layers such as the drag coefficient $\left(C_{\mathrm{d}}\right)$ and the recirculation length $\left(L_{\mathrm{r}} / D\right)$, the DES1 results based on the fine grid provide an underestimated $C_{\mathrm{d}}$ and excessively longer $L_{\mathrm{r}} / D$ with undesirable longer shear layers, and this result is consistent with that of [50], whereas both the ISAS and SAS results are in excellent agreement with the measurements, mainly benefiting from the gridindependent $L_{\mathrm{vk}}$ scale which will not introduce unpredictable efforts into the detachment mechanism of the shear layers. When using the coarse grid, the $C_{\mathrm{d}}$ and $L_{\mathrm{r}} / D$ obtained from ISAS2 are still closer to the measurements, while the results of DES and SAS are comparable with each other and the
DES model gives a more accurate recirculation length. As for the minimum back flow velocity $\left(-U_{\min } / U_{\infty}\right)$, the ISAS results are not the best compared with DES and SAS results but still comparable with most of numerical references, while there is minor difference between the DES and SAS results. As for the base pressure coefficient $\left(-C_{\mathrm{pb}}\right)$, the ISAS results are better than the other two simulations, while between the DES and SAS results, it is still shown that each of them is better on the fine or coarse grids. Finally, the discrepancy from the three models is very small concerning the Strouhal number (St) and the separation angle $\left(\theta_{\text {sep }}\right)$, and all results are comparable with the other numerical results.

The capabilities of current models in reproducing turbulent unsteadiness are displayed in Figure 13, which shows the instantaneous turbulent structures identified by the $Q$-criterion iso-surface and coloured by velocity magnitude. As observed in Figures 13(a)-13(c), all the three models 


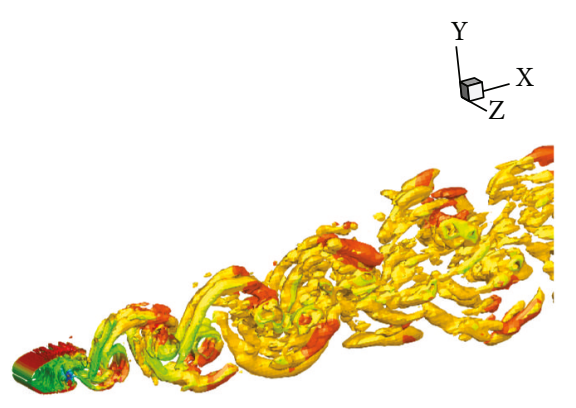

(a) ISAS1

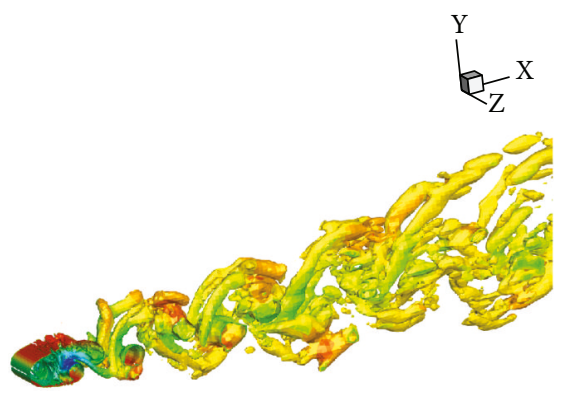

(c) SAS1

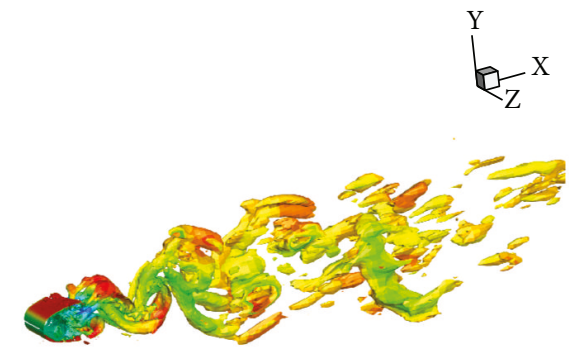

(e) DES2

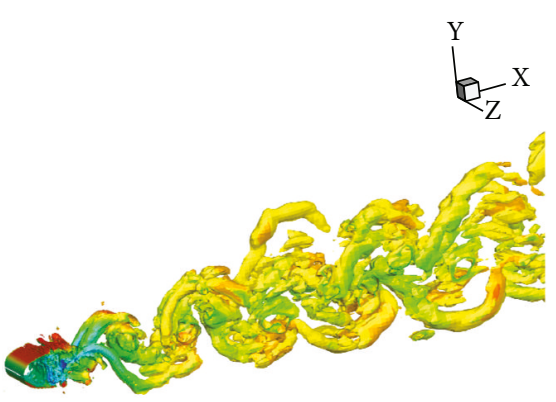

(b) DES1

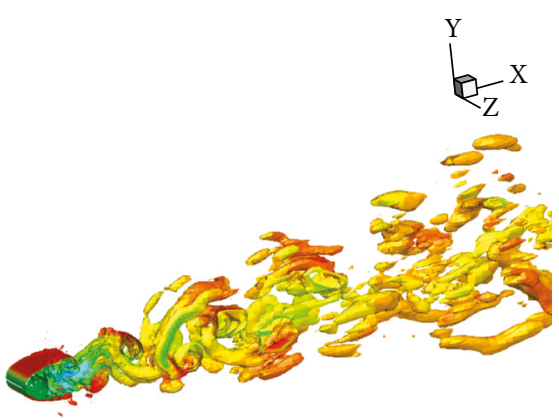

(d) ISAS2

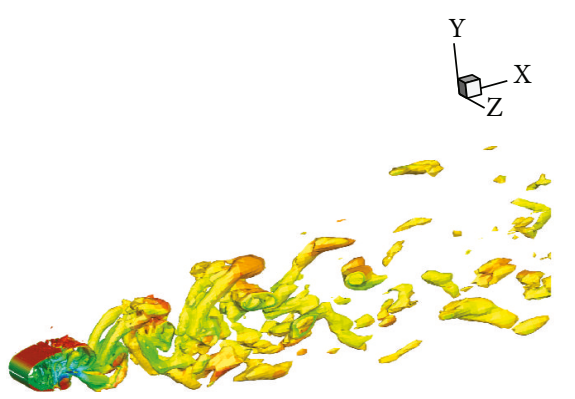

(f) SAS2

FIgURE 13: Instantaneous turbulent structures identified by the Q-criterion.

fairly represent the stably two-dimensional boundary layer and three-dimensional structures with LES-like modifications, while the coarse grid does not allow the same spatial resolution as the fine one, resulting in obviously rougher turbulent structures, shown in Figures 13(d) and 13(e). Through closer inspection, it shows that the ISAS model captures the dynamically smaller structures as well as DES model and better than SAS with higher viscosity level and excessive dissipation.

Finally, the one-dimensional energy spectrum is plotted in Figure 14, which is obtained through the analysis to the transverse velocity at $x / D=3$ line of the wake. Noting that the frequency in $x$-axis is normalized by the characteristic frequency $f_{\mathrm{vs}}\left(f_{\mathrm{vs}}=\mathrm{St} U_{\infty} / D\right)$, all the three models allow the formulation of turbulent spectra and capture the two peaks very well. Though the smaller turbulent structures with low energy and high frequency have been decayed under current grid limit, all the plots fairly match the $-5 / 3$ slope relevant to Kolmogorov's energy spectrum, enabling the models to simulate the larger structures with high energy and low frequency.

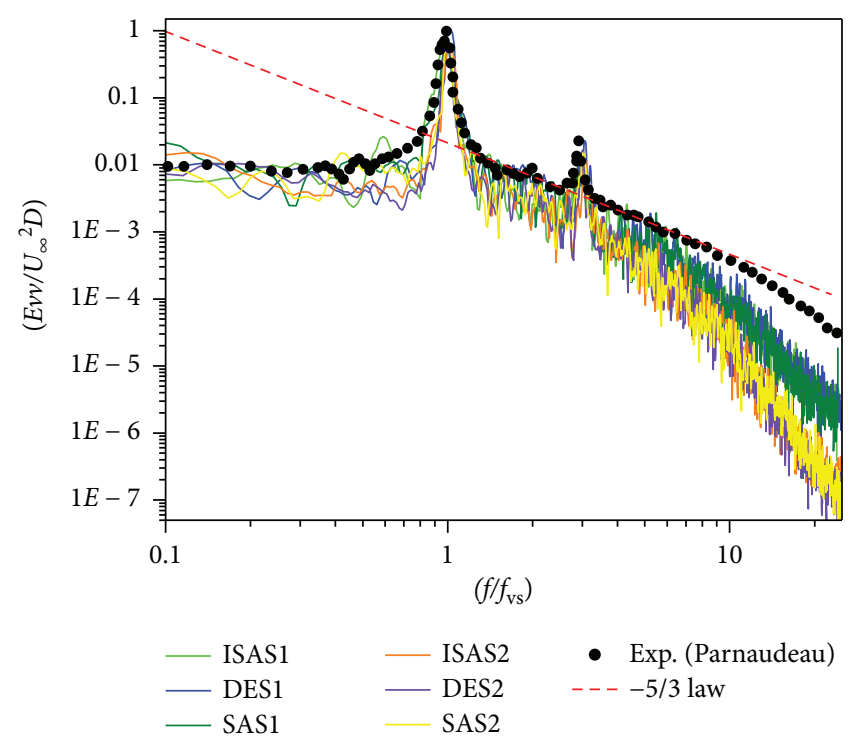

FIgURE 14: One-dimensional spectra of the transverse velocity at $x / D=3$. 


\section{Summary and Conclusions}

In this paper, by introducing the von Karman factor into the scale-determining equation, a new hybrid turbulent model combining the features of DES and SAS methods is proposed for massively separated flows, and with the concept of scale-adaptive, the underlying method is termed improved scale-adaptive simulation (ISAS). The derivation and calibration of the transformed formulation are presented, and the cylinder flow at $\mathrm{Re}=3900$ is selected as the benchmark case for the model validation. Detailed numerical comparisons with the available experimental data are conducted to study the properties of the proposed ISAS model. As expected, the ISAS model is capable to simulate turbulent details down to the grid limit in a simple and efficient way and, furthermore, appears some advantages over the original DES and SAS models.

From the analysis to the energy spectrum of the freely decaying isotropic homogeneous flow and the cylinder flow, it is shown that the ISAS model allows for the turbulent spectra close to the Kolmogorov's energy spectrum, demonstrating the capability of which in representing the resolvable contents with lower frequency.

By comparison with DES model, with similar formulation, the efficiency of ISAS in providing a convergent simulation is very close to that of DES. The $L_{\mathrm{vk}}$ length scale, according to the local flow physics, enables ISAS model to dynamically adjust the resolution to resolved spectra and appear comparable with DES in capturing the transient unsteadiness. Meanwhile, benefiting from the gridinsensitive character of $L_{\mathrm{vk}}$ scale, it enables ISAS model to operate a smooth switch from stable mode to unstable mode and avoid the grid-dependent issues such as gray area and modeled stress depletion appeared in DES simulations.

For the comparison with SAS model, with the reformulation in ISAS, the concept of scale-adaptive is made more intuitive and efficient than SAS. The resolving speed of SAS is noticeably improved through the simplified process. With a more flexible adjusting mechanism, the ISAS model provides a more feasible dissipative level and captures finer turbulent structures than SAS.

Concerning the benchmark cylinder flow, with an applicable viscosity level, the development of the shear layers is reproduced very well by the ISAS model. Thus, the focused quantities of industrial interest such as the drag coefficient and recirculation length are in the best agreement with the experimental data on fine grid. All the numerical accuracy from the coarse grid is reasonably decreased for the comparative ISAS, DES, and SAS models, though not always the best such as the minimum back flow velocity and Reynolds stress, most of the ISAS outputs are still closer to the measurements.

In general, the establishment of ISAS is a positive attempt to the combination and remedy of current hybrid methods considering the still unaffordable LES situation. The proposed method allows for a good compromise regarding the numerical accuracy, efficiency, and robustness, which is beneficial for the development of turbulence models with practical value for the engineering flows.

\section{Conflicts of Interest}

The authors declare that they have no conflicts of interest.

\section{Acknowledgments}

The authors wish to acknowledge the National Natural Science Foundation of China, Award no. 11202101.

\section{References}

[1] M. R. Malik and D. M. Bushnell, "Role of computational fluid dynamics and wind tunnels in aeronautics R\&D," US Patent TP-2012-217602, 2012.

[2] J. Slotnick, A. Khodadoust, D. Darmofal, W. Gropp, E. Lurie, and D. Mavriplis, "CFD vision 2030 study: a path to revolutionary computational aerosciences," US Patent CR-2014218178, 2014.

[3] J. Fröhlich and D. von Terzi, "Hybrid LES/RANS methods for the simulation of turbulent flows," Progress in Aerospace Sciences, vol. 44, no. 5, pp. 349-377, 2008.

[4] U. Piomelli, "Large eddy simulations in 2030 and beyond," Philosophical Transactions of the Royal Society A: Mathematical, Phisical and Engineering Sciences, vol. 372, no. 2022, article 20130320, 2014.

[5] C. D. Argyropoulos and N. C. Markatos, "Recent advances on the numerical modelling of turbulent flows," Applied Mathematical Modelling, vol. 39, no. 2, pp. 693-732, 2015.

[6] P. Spalart, M. Strelets, and S. Allmaras, "Comments on the feasibility of LES for wings, and on a hybrid RANS/LES approach," in Paper Presented at the Meeting of the First AFOSR International Conference on DNS/LES, Colombus, OH, USA, 1997.

[7] G. Kumar, S. K. Lakshmanan, H. Gopalan, and A. De, "Investigation of the sensitivity of turbulent closures and coupling of hybrid RANS-LES models for predicting flow fields with separation and reattachment," International Journal for Numerical Methods in Fluids, vol. 83, no. 12, pp. 917-939, 2017.

[8] D. Li, "Numerical simulation of thin airfoil stall by using a modified DES approach," International Journal for Numerical Methods in Fluids, vol. 54, no. 3, pp. 325-332, 2007.

[9] S. Tu, S. Aliabadi, R. Patel, and M. Watts, "An implementation of the Spalart-Allmaras DES model in an implicit unstructured hybrid finite volume/element solver for incompressible turbulent flow," International Journal for Numerical Methods in Fluids, vol. 59, no. 9, pp. 1051-1062, 2009.

[10] P. R. Spalart, "Detached-eddy simulation," Annual Review of Fluid Mechanics, vol. 41, no. 1, pp. 181-202, 2009.

[11] P. R. Spalart, S. Deck, M. L. Shur, K. D. Squires, M. K. Strelets, and A. Travin, "A new version of detached-eddy simulation, resistant to ambiguous grid densities," Theoretical and Computational Fluid Dynamics, vol. 20, no. 3, pp. 181-195, 2006.

[12] M. L. Shur, P. R. Spalart, M. K. Strelets, and A. K. Travin, "A hybrid RANS-LES approach with delayed-DES and wallmodelled LES capabilities," International Journal of Heat and Fluid Flow, vol. 29, no. 6, pp. 1638-1649, 2008.

[13] M. S. Gritskevich, A. V. Garbaruk, J. Schütze, and F. R. Menter, "Development of DDES and IDDES formulations for the $k-\omega$ shear stress transport model," Flow, Turbulence and Combustion, vol. 88, no. 3, pp. 431-449, 2012. 
[14] Z. Xiao, J. Liu, K. Luo, J. Huang, and S. Fu, "Investigation of flows around a rudimentary landing gear with advanced detached-eddy-simulation approaches," AIAA Journal, vol. 51, no. 1, pp. 107-125, 2013.

[15] L. H. Xiao, Z. X. Xiao, Z. W. Duan, and S. Fu, "Improveddelayed-detached-eddy simulation of cavity-induced transition in hypersonic boundary layer," International Journal of Heat and Fluid Flow, vol. 51, pp. 138-150, 2015.

[16] W. L. Zheng, C. Yan, H. K. Liu, and D. H. Luo, "Comparative assessment of SAS and DES turbulence modeling for massively separated flows," Acta Mechanica Sinica, vol. 32, no. 1, pp. $12-$ 21, 2016.

[17] F. R. Menter, M. Kuntz, and R. Langtry, "Ten years of industrial experience with the SST turbulence model," Turbulence Heat and Mass Transfer, vol. 4, 2003.

[18] F. R. Menter, M. Kuntz, and R. Bender, "A scale-adaptive simulation model for turbulent flow predictions," in 41st Aerospace Science Meeting and Exhibit, Reno, NV, USA, 2003.

[19] F. R. Menter and Y. Egorov, "A scale adaptive simulation model using two-equation models," in 43rd AIAA Aerospace Sciences Meeting and Exhibit, Reno, NV, USA, January 2005.

[20] F. R. Mentor and Y. Egorov, "The scale-adaptive simulation method for unsteady turbulent flow predictions. Part 1: theory and model description," Flow, Turbulence and Combustion, vol. 85, no. 1, pp. 113-138, 2005.

[21] Y. Egorov and F. R. Menter, "Development and application of SST-SAS turbulence model in the DESIDER project," in Advances in Hybrid RANS-LES Modelling, S. H. Peng and W. Haase, Eds., vol. 97 of Notes on Numerical Fluid Mechanics and Multidisciplinary Design, pp. 261-270, Springer, Berlin, Heidelberg, 2008.

[22] Y. Egorov, F. R. Mentor, R. Lechner, and D. Cokljat, "The scale-adaptive simulation method for unsteady turbulent flow predictions. Part 2: application to complex flows," Flow, Turbulence and Combustion, vol. 85, no. 1, pp. 139-165, 2010.

[23] L. Davidson, "Evaluation of the SST-SAS model: channel flow, asymmetrical diffuser and axisymmetric hill," in European Conference on Computational Fluid Dynamics, TU Delft, The Netherlands, 2006.

[24] M. Kaltenbacher, M. Escobar, S. Becker, and I. Ali, "Numerical simulation of flow-induced noise using LES/SAS and Lighthill's acoustic analogy," International Journal for Numerical Methods in Fluids, vol. 63, no. 9, pp. 1103-1122, 2010.

[25] P. Spalart, "Young-Person's guide to detached-eddy simulation grids," US Patent NASA/CR-2001-211032, 2001.

[26] S. R. Bruno, N. Bertier, S. Deck, and F. Dupoirieus, "A DES method applied to a backward facing step reactive flow," Comptes Rendus Mecanique, vol. 337, no. 6-7, pp. 340-351, 2009.

[27] R. Z. Gao, J. L. Xu, R. Zhao, and C. Yan, "Evaluation of XY-SAS model for separated flows," Journal of Beingjing University of Aernnautics and Astronauticss, vol. 36, no. 4, pp. $415-419,2010$.

[28] C. Y. Xu, T. Zhou, C. L. Wang, and J. H. Sun, “Application of scale-adaptive simulation technique based on one-equation turbulence model," Applied Mathematics and Mechanics, vol. 36, no. 1, pp. 121-130, 2015.

[29] A. Travin, M. Shur, P. Spalart, and M. Strelets, "On URANS solutions with LES-like behaviour," in Proceedings of
ECCOMAS congress on Computational Methods in Applied Science and Engineering, Jyväskylä, Finland, July 2004.

[30] F. R. Menter, "Two-equation eddy-viscosity turbulence models for engineering applications," AIAA Journal, vol. 32, no. 8, pp. 1598-1605, 1994.

[31] F. R. Menter, "Review of the shear-stress transport turbulence model experience from an industrial perspective," International Journal of Computational Fluid Dynamics, vol. 23, no. 4, pp. 305-316, 2009.

[32] M. Strelets, "Detached eddy simulation of massively separated flows," in 39th AIAA Aerospace Sciences Meeting and Exhibit, Reno, NV, USA, 2001.

[33] F. R. Menter and Y. Egorov, "Revisiting the turbulent scale equation," in IUTAM Symposium on One Hundred Years of Boundary Layer Research, 129, Solid mechanics and its applications, G. E. A. Meier, K. R. Sreenivasan, and H. J. Heinemann, Eds., Springer, Dordrecht, Netherlands, 2006.

[34] L. Du and F. F. Ning, "Scale adaptive simulation of flows past an airfoil after stall," in ASME 2012 Fluids Engineering Division Summer Meeting collocated with the ASME 2012 Heat Transfer Summer Conference and the ASME 2012 10th International Conference on Nanochannels, Microchannels, and Minichannels, vol. 1 of Symposia, Parts $A$ and B, pp. 1551-1560, Rio Grande, PR, USA, July 2012.

[35] G. Comtebellot and S. Corrsin, "Simple Eulerian time correlation of full-and narrow-band velocity signals in grid generated, 'isotropic' turbulence," Journal of Fluid Mechanics, vol. 48, no. 2, pp. 273-337, 1971.

[36] N. F. Ducros, "Subgrid-scale stress modelling based on the square of the velocity gradient tensor," Flow, Turbulence and Combustion, vol. 62, no. 3, pp. 183-200, 1999.

[37] D. H. Luo, C. Yan, H. K. Liu, and R. Zhao, "Comparative assessment of PANS and DES for simulation of flow past a circular cylinder," Journal of Wind Engineering and Industrial Aerodynamics, vol. 134, pp. 65-77, 2014.

[38] A. G. Kravchenko and P. Moin, "Numerical studies of flow over a circular cylinder at $\operatorname{Re}_{D}=3900$," Physics of Fluids, vol. 12, no. 2, pp. 403-417, 2000.

[39] C. J. Doolan, "Large eddy simulation of the near wake of a circular cylinder at sub-critical Reynolds number," Engineering Applications of Computational Fluid Mechanics, vol. 4, no. 4, pp. 496-510, 2010.

[40] V. D. Alessandro, S. Montelpare, and R. Ricci, "Detached-eddy simulations of the flow over a cylinder at $R e=3900$ using OpenFoam," Computers \& Fluids, vol. 136, pp. 152-169, 2016.

[41] D. A. Lysenko, I. S. Ertesvag, and K. E. Rian, "Large-eddy simulation of the flow over a circular cylinder at Reynolds number $2 \times 10^{4}$," Flow Turbulence and Combustion, vol. 92, no. 3, pp. 673-698, 2014.

[42] H. Jasak, H. G. Weller, and A. D. Gosman, "High resolution NVD differencing scheme for arbitrarily unstructured meshes," International Journal for Numerical Methods in Fluids, vol. 31, no. 2, pp. 431-449, 1999.

[43] J. Franke and W. Frank, "Large eddy simulation of the flow past a circular cylinder at $R e_{D}=3900$," Journal of Wind Engineering and Industrial Aerodynamics, vol. 90, no. 10, pp. 1191-1206, 2002.

[44] P. Parnaudeau, J. Carlier, D. Heitz, and E. Lamballais, "Experimental and numerical studies of the flow over a circular cylinder at Reynolds number 3900," Physics of Fluids, vol. 20, article 085101, 2008. 
[45] L. Ong and J. Wallace, "The velocity field of the turbulent very near wake of a circular cylinder," Experiments in Fluids, vol. 20, no. 6, pp. 441-453, 1996.

[46] C. Norberg, Effects of Reynolds Number and a Low-Intensity Free-Stream Turbulence on the Flow around a Circular Cylinder, Department of Applied Thermodynamics and Fluid Mechanics: Publication No. 87/2, Chalmers University of Technology, Sweden, 1987.

[47] X. Ma, G. S. Karamanos, and G. E. Karniadakis, "Dynamics and low-dimensionality of a turbulent near wake," Journal of Fluid Mechanics, vol. 410, pp. 29-65, 2000.

[48] S. Dong, G. E. Karniadakis, A. Ekmekci, and D. Rockwell, “A combined direct numerical simulation-particle image velocimetry study of the turbulent near wake," Journal of Fluid Mechanics, vol. 569, pp. 185-207, 2006.

[49] S. Jee and K. Shariff, "Detached-eddy simulation based on the $v^{2}-f$ model," in Seventh International Conference on Computational Fluid Dynamics, Big Island, Hawaii, July 2012.

[50] Y. Zhang, W. G. Habashi, and R. Khurram, "Zonal detachededdy simulation of turbulent unsteady flow over iced airfoils," Journal of Aircraft, vol. 53, no. 1, pp. 1-14, 2016. 


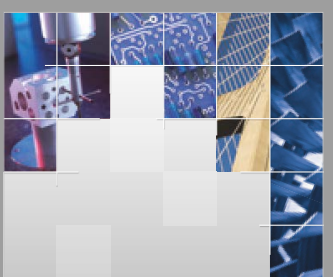

\section{Enfincering}
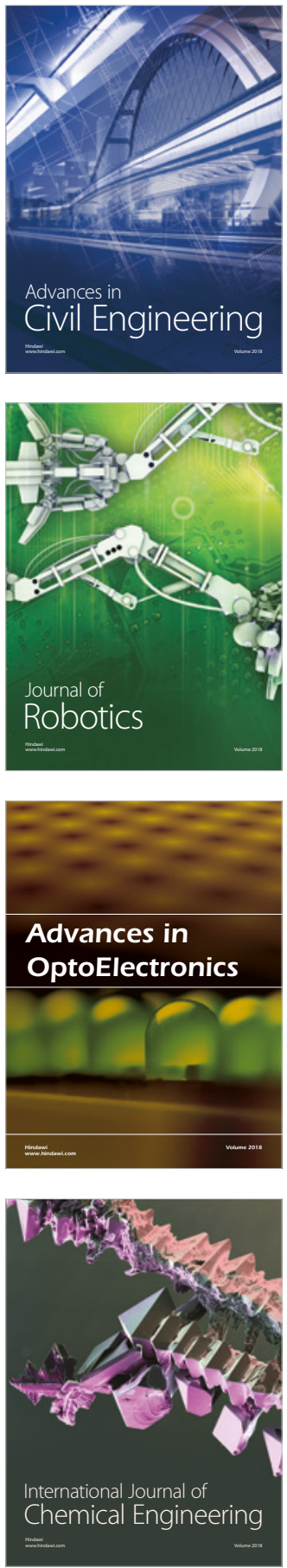

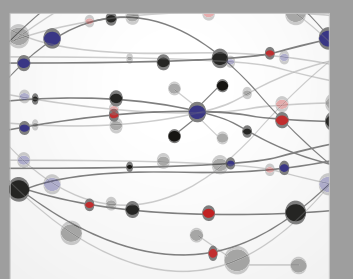

\section{Rotating \\ Machinery}

The Scientific World Journal

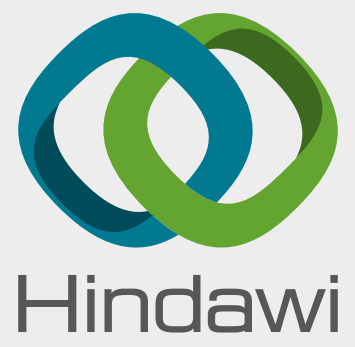

Submit your manuscripts at

www.hindawi.com
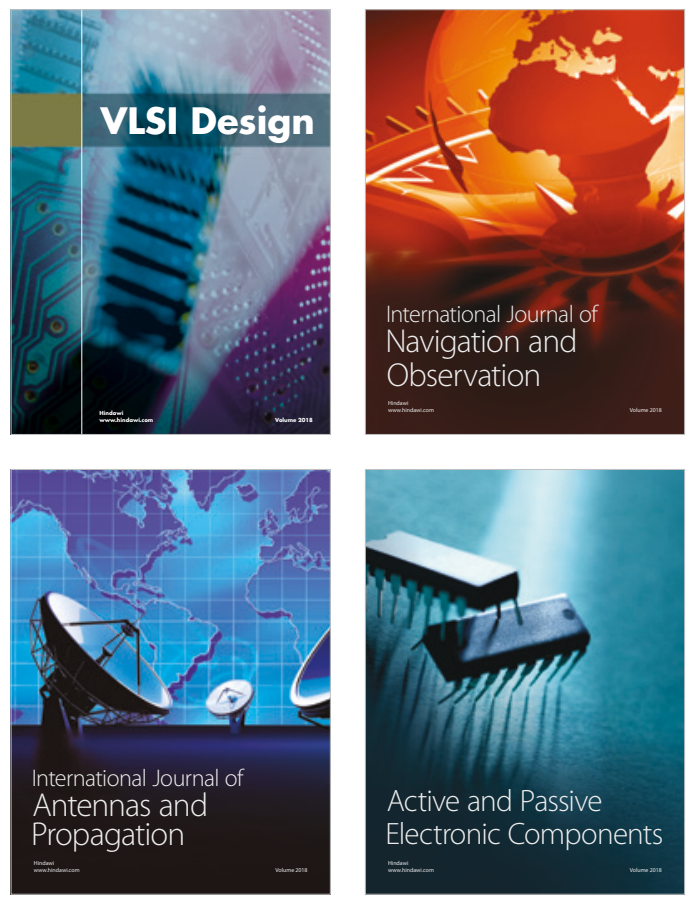
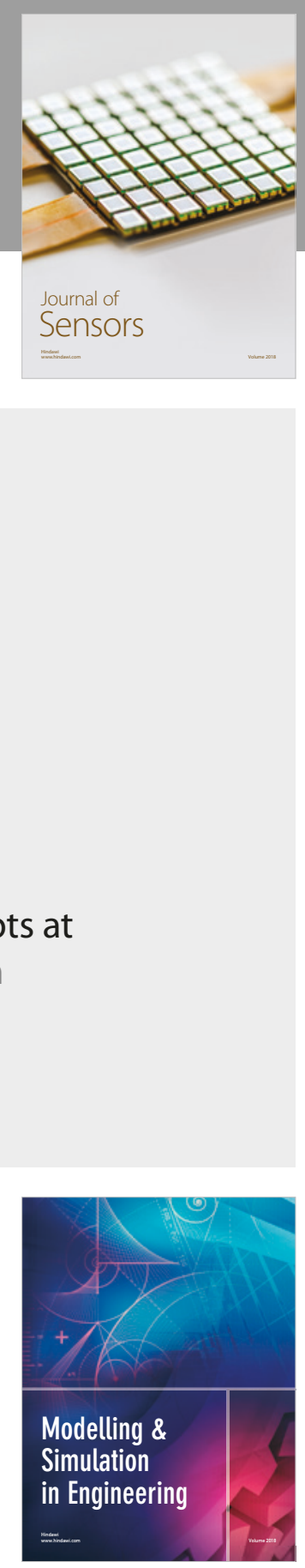

\section{Advances \\ Multimedia}
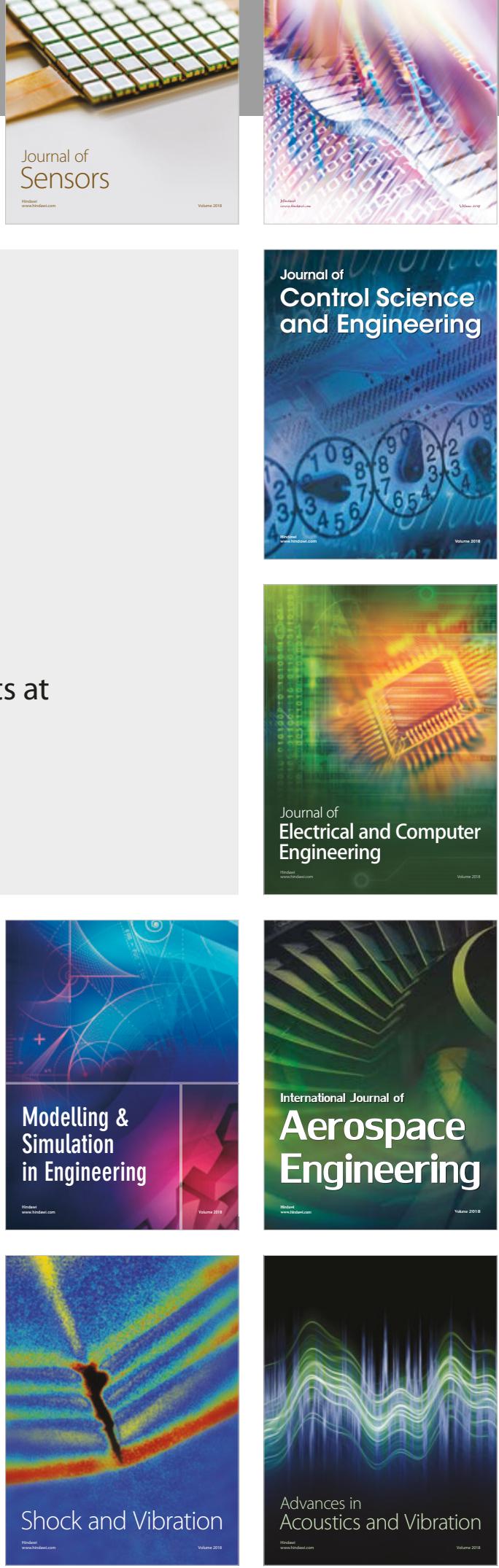